\title{
Weitere Studien über die von einzelnen Organen her- vorgebrachten Substanzen mit spezifischer Wirkung ${ }^{1}$ ).
}

\author{
II. Mitteilung ${ }^{2}$ ). \\ Fon \\ Emil Abderhalden.
}

(Aus dem physiologischen Inștitut der Universität Halle.)

Mit Tafel III-VI.

(Lïngegangen am 5. Mai 1919.)

Die Forschungen der letzten Jahre haben immer mehr Material für die Annahme erbracht, dass der gesamte Stoffwechsel in Abhängigkeit von bestimmten exogenen Nahrungsstoffen steht. Je tiefer in das ganze Forschungsgebiet vorgedrungen wird, um so mehr zeigt es sich, dass die Verhältnisse nicht einfach liegen. Schon der Umstand, dass die einzelnen Tierarten auf das Fehlen bestimmter Stoffe ganz verschieden antworten, und ferner die Ausfallserscheinungen nicht durch dieselben Stoffe zu beheben sind, zeigt, dass von einer Vielheit von Substanzen gesprochen werden muss. Mir scheint immer mehr die Annahme am wahrscheinlichsten. dass man die sogenannten Nutramine als Reizstoffe aufzufassen hat, auf die bestimmte Zellarten im Organismus eingestellt sind. Es liegt in gewissem Sinne eine Wechselbeziehung vor, die über den Zellstaat des einzelnen Individuums hinaus sich auf die Aussenwelt erstreckt. Vielleicht handelt es sich im einzelnen Falle um eine Anpassungserscheinung. Mit der Nahrung werden diese Stoffe normalerweise aufgenommen. Sie begleiten die einzelnen Nahrungsstoffe und sind vielleicht notwendig, damit deren Verwendung im Organismus in die richtigen Bahnen gelenkt wird. Allem Anschein nach wirken diese Stoffe, oder viele davon, auf die Verdauungsdrüsen ein. Ferner ist ein Finfluss auf die Darmperistaltik unverkennbar. Darüber hinaus sind ohne

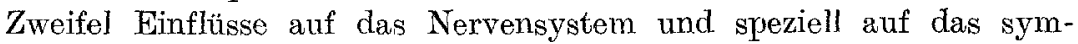
pathische erkennbar.

Das ganze erwähnte Forschungsgebiet steht ohne Zweifel in engsten Beziehungen zu dem Problem der Wechselbeziehungen der

1) Ausgeführt mit Mitteln der Kaiser Wilhelm-Gesellschaft.

2) I. Mitteilung: Dieses Archiv Bd. 162 S. 99.1915 . 
Weit. Studien üb. die von einz. Organen hervorgebrachten Substanzen. II. 237

einzelnen Organe zueinander. Zurzeit stellen wir uns vor, dass. die einzelnen Zellarten Stoffe hervorbringen, die ausserhalb des Zellverbandes in anderen Zellarten bestimmte Funktionen in iie Wege leiten oder doch beeinflussen. Wir nehmen an, dass sogenannte Inkrete der Lymphbahn oder Blutbahn übergeben werden, worauf sie dann eine Fernwirkung dadurch entfalten können, dass sie zu bestimmten Zellen in Beziehung treten. Wir dürfen uns diese Beziehungen nicht zu einseitig vorstellen. Sie brauchen nicht nur chemischer Natur zu sein. Es ist sehr wohl möglich, dass physikalische und physikalischchemische Einflüsse sich geltend machen. Vor allen Dingen muss man an Beziehungen zu den Kolloiden der Zellen denken. Vor allem wäre es möglich, dass durch die Inkrete auch die Durchlässigkeit der sogenannten Zellmembranen, das heisst der Grenzschicht in den Zellen in typischer Weise beeinflusst würde. Überblickt man das ganze grosse Forschungsgebiet rein sachlich, dann muss man anerkennen, dass zurzeit die Hypothesen noch eimen sehr weiten Spielraum haben, während das tatsächlich Festgestellte noch einen kleinen Raum einnimmt. Um so mehr muss es unser Bestreben sein, aus den einzelnen Organen Stoffe zu isolieren, die rein sind und ganz bestimmte Wirkungen entfalten. Wahrscheinlich wird sich dabei herausstellen, dass der Organismus nicht mit einzeInen Stoffen, sondern mit Gemengen von solchen arbeitet. Vor derartigen Studien schreckte die Vorstellung zurück, dass die Inkrete hochmolekulare, kompliziert gebaute Stoffe sein sollten. Dieser Gedanke wurde immer wieder genährt, weil bestimmt behauptet wurde, dass zum Beispiel das wirksame Prinzip der Schilddrüse ein jodhaltiger Eiweisskörper sei.

Da nun die Schilddrüse auch dann bestimmte Wirkungen im Organismus hervorbringt, wenn sie verfüttert wird, so musste der Verdacht geweckt werden, dass vielleicht doch die wirksamen Stoffe - die sogenannten Inkrete - einfacherer Natur sein könnten, als man bis jetzt angenommen hatte. Dieser Gedankengang veranlasste meine Studien über das Verhalten von vollständig abgebauten Organen gegenüber verschiedenen Funktionen im Organismus höherer Tiere und. auf das Wachstum und die Entwicklung verschiedener niederer Tierarten. Der Plan war vollständig klar vorgezeichnet. Zunächst musste festgestellt werden, ob gänzlich eiweiss- und peptonfreie Organbestandteile noch irgendwelche spezifische Wirkungen haben. Der Abbau. wurde zunächst durch Autolyse vollzogen. Dabei erhält man allerdings keine vollständige Spaltung. Dieser Weg wurde beschritten, um fremdartige Zusätze zu vermeiden. In der Folge wurde dann der Abbau der einzelnen Organe so durchgeführt, dass Autolyse und Verdauung mit Pankreas- und Darmsaft zusammen durchgeführt wurden. In einigen Fällen wurde die Verdanung durch Magensaft eingeleitet. 
Von Zeit zu Zeit wurden die Verdauungssäfte von neuem zugesetzt. Gewöhnlich dauerte die einzelne Verdauung 1-2 Monate. Das Fortschreiten des Abbanes wurde an Hand der Biuretreaktion kontrolliert. Zur Kontrolle wurden stets Versuche ausgeführt, bei denen die unverärderten Organe direkt verwendet wurden.

Bei späteren Versuchen wurden die Organe mit Säure abgebaut. Dieser Weg wurde nicht sofort beschritten, weil die Erfahrung gezeigt hat, dass zahlreiche Stoffe das Kochen mit Säuren nicht vertragen. Der Abbau durch Fermente ist auf alle Fälle der mildere. Der Abbau mit Säure wurde in folgender Weise durchgeführt: Die Organe wurden blutfrei gewaschen und dann ganz fein zerkleinert. Nunmehr wurden sie auf Filtrierpapier vom aussen anhaftenden Wasser befreit und dann mit der zehnfachen Menge 5\% iger Schwefelsäure bei der Temperatur: des Wasserbades so lange gekocht, bis vollständige Lösung eingetreten war. Im Durchsehnitt musste vier- bis sechsmal 8 Stunden gekocht werden. Später wurde die Schwefelsäure $10 \%$ ig angewandt. Das Hydrolysat wurde filtriert und die Schwefelsäure sehr sorgsam mit Baryt entfernt. Hierbei wurde peinlich genau darauf geachtet, dass die Reaktion niemals alkalisçch wurde. Die schwefelsäure- und barytfreie Lösung wurde vom Bariumsulfat durch Filtrieren getrennt und dann bei $40^{\circ}$ des Wasserbades und einem Druck von ungefähr $15 \mathrm{~mm} \mathrm{Hg}$ zur Trockene verdampft. Der Rückstand wurde in Wasser gèlöst und dann zu den Versuchen verwendet.

In einigen Fällen wurden die Organe, bevor sie hydrolysiert wurden, mit verschiedenen Lösungsmitteln, wie Alkohol, Aceton, Chloroform, ausgezogen und die Extrakte für sich geprüft. In anderen Fällen wurde der oben erwähnte Destillationsrückstand, der die Spaltprodukte aus den Organen enthielt, mit den gleichen Lösungsmitteln behandelt. Ferner wurden Fällungen erzengt, das heisst, die alkoholische Lösung wurde zum Beispiel mit Aceton gefällt. Niederschlag und nichtgefällter Anteil wurden dann getrennt auf Wirksamkeit geprüft. Diesen Versuchen kommt zunächst keine ausschlaggebende Bedeutung zu, denn die Erfahrung hat gezeigt, dass unreine Stoffe sehr leicht andere mit niederreissen oder aber in Lösung halten. Von einer reinlichen Trennung kann keine Rede sein. Ja, gleich durchgeführte Trennungsmethoden können, wie der direkte Versuch zeigt, unter Umständen zu ganz verschiedenen Resultaten führen. Wir legen deshalb auf die aus derartigen Versuchen gezogenen Schlussfolgerungen keinen allzu grossen Wert.

Vor allen Dingen müsste in jedem einzelnen Falle geprüft werden, ob eine isolierte Substanz, die sich als unwirksam erweist, auch im genuinen Zustande diese Eigenschaft besitzt, oder aber erst durch die ganze Behandlungsmethode unwirksam geworden ist. Erweist es sich 
Weit. Studien üb. die von einz. Organen hervorgebrachten Substanzen. II. 239

ferner, dass aus einem Gemisch abgetrennte Substanzen andere Wirkungen zeigen als dieses, dann muss ebenfalls festgestellt werten, ob nicht die angewandte Methode schuld an der ganzen Veränderung ist. Man darf nicht einfach mit allen möglichen Lösungsmitteln trennen und sich damit begnügen, die besonderen Wirkungen der einzelnen Fraktionen zu prüfen. Die gegebene Versuchsordnung für die Entscheidung derartiger Fragestellungen ist die folgende: Hat man eine Substanz, die sich als wirksam erweist, und gelingt es, aus thr eine unwirksame und eine wirksame Komponente abzutrennen, von denen die letztere eine andere Wirkung hat als das Gemisch, dann muss festgestellt werden, ob nach Vercinigung dor getrennten Komponenten wieder die ursprüngliche Wirkung zustande kommt, oder aber, ob durch die angewandte Methode sich eine Veränderung einer oder beider Komponenten nachweisen lässt. Solange nur die abgetrennten Produkte für sich untersucht werden, wird man nie zu klaren Schlüssen kommen. Wir teilen deshalb unsere reichlichen, nach dieser Richtung ausgeführten Versuche noch nicht mit, weil sie von dem erwähnten Gesichtspunkte aus noch nicht spruchreif sind.

Die von mir gefundene Tatsache, dass vollständig eiweissfreie, tief abgebaute Organe qualitativ die gleiche Wirkung auf das Wachstum und die Entwicklung von Kaulquappen ausüben, wie die nicht abgebauten Gewebe, ist von J. M. Rogoff und David Marine ${ }^{1}$ ) und Romeis ${ }^{2}$ ) vollständig bestätigt worden. Auch diese Forscher konnten zeigen, dass hydrolysierte Organe in der genannten Beziehung wirksam sind. 'Romeis hat darüber hinaus versucht, bestimmte Stoffe aus den Organen zu isolieren, und die Frage zu entscheiden, ob bestimmte Körperklassen für die Wirkung verantwortlich gemacht werden können. Er hat damit den gleichen Versuchsplan betreten, den ich auf breiter Grundlage durchführen wollte. Selbstverständlich hat jeder Forscher auf dem ursprünglich von Gudernatsch eröfneten Forschungsgebiete freie Bahn. Ich würde die folgenden Versuchsergebnisse gern noch zurückgehalten haben, um im Laufe der Zeit zu einem abgerundeten Ergebnis zu kommen. Die von Romeis mitgeteilten Untersuchungen veranlassen mich jedoch, jetzt schon diejenigen Resultate mitzuteilen, die in sich abgeschlossen sind.

Es sei auch darauf hingewiesen, dass Abelin ${ }^{3}$ ) die wichtige Beobachtung gemacht hat, dass ein eiweissfreies jodarmes Schilddrüsenpräparat ebeńso auf den Stoffwechsel wirkte, wie ein eiweisshaltiges,

1) J. M. Rogoff und David Marine, J. Pharm. Therap. Bd. 9 S. 57. 1916.

2) Benno Romeis, Z. f. die gesamte experim. Medizin. 6. und Dieses Archiv Bd. 173 S. 422.1919.

3) J. A belin, Biochem. Zeitschr. Bd. 80 S. 259. 1917.

Pflüger's Arehiv für Physiologie. Bd. 176 . 
das heisst, auch er hat feststellen können, dass die Wirkung der Schilddrüse nicht an das Vorhandensein eines jodhaltigen Eiweisskörpers geknüpft ist.

Es unterliegt keinem Zweifel, dass die Inkretionsorgane geringe Inkretmengen in freiem Zustand in sich enthalten. Laugt man frische Organe mit Wasser aus, so erhält man in ihm wirksame Stoffe. Ihre Menge ist jedoch sehr gering. Sie steigt mit zunehmender Autolyse. Wird ein Organ durch Auskochen mit Wasser von allen löslichen Bestandteilen befreit, dann gewinnt man bei seiner Hydrolyse wieder wirksame 'Stoffe. Es geht daraus hervor, dass die Inkretstoffe in den Organen zum kleinsten Teil frei, zum grössten Teil in Bindung vorhanden sind.

Ich möchte noch ganz besonders hervorheben, dass die ausgeführten Untersuchungen auf keinen Fall etwas über die gesamte Funktion der geprüften Organe aussagen können. Es werden ja immer nur Teilfunktionen geprüft, so zum Beispiel Einflüsse auf das Wachstum und die Metamorphose von niederen Tieren, oder es wird der Einfluss auf die glatte Muskulatur für sich festgestellt. Selbstverständlich kann ein bestimmter Einfluss beobachtet oder aber vermisst werden, und trotzdem kann das geprüfte Produkt in anderer Beziehung unwirksam oder aber wirksam sein. Man kann somit einstweilen auch nicht die gesamte pharmakologische Prüfung der einzelnen Inkrete, zum Beispiel durch Kaulquappenversuche, ersetzen. Es ist von grösster Bedeutung, dass die einzelnen Stoffe auch am höher organisierten Tiere und vor allen Dingen an überlebenden Organen geprüft werden. Te mehr verschiedenartige Beobachtungen vorliegen, um so weiter wird unser Blick für die Beurteilung der Funktionen der einzelnen Organe werden.

Meine eigenen Studien sind in der Hauptsache in den Jahren 1914 bis 1918 ausgeführt worden. Sie werden auch in diesem Jahre fortgesetzt. Es sei ausdrücklich hervorgehoben, dass es notwendig ist. die Zahl der Versuche möglichst gross zu gestalten und vor allen Dingen möglichst viele Kontrollversuche durchzuführen. Es hat sich gezeigt, dass auch ohne Zusatz von besonderen. Substanzen eigenartige Erscheinungen im Wachstum und in der Metamorphose vorkommen. Unter 1000 Kaulquappen waren immer 10-20, die in dieser Richtung Abnormitäten zeigten. Wir haben beschleunigte Metamorphosen beobachtet, das heisst, es bekamen Kaulquappen innerhalb der ersten 10 bis 14 Tage alle vier Beine, während die überwiegend grosse Mehrzahl der Versuchstiere unter denselben Bedingungen ihre Metamorphose beträchtlich später vollendete. Die sich rasch entwickelnden Tiere blieben klein. Sie waren meist auch heller gefärbt. Wieder andere zeigten ein gesteigertes Wachstum, oder besser ausgedrückt, 
Weit. Studien ảb. die von einz. Organen hervorgebrachten Substanzen. II. 241

ihre Körpergrösse nahm bedeutend zu, während die Metamorphose im Rückstand blieb. Es wird von grösstem Interesse sein, festzustellen, ob diesen Erscheinungen Anomalien in der Funktion der Schilddrüse und der Thymusdrüse oder anderer Organe zugrunde liegen. Histologische Untersuchungen sind bereits im Gange. Bemerkt sei noch, dass es vorkommen kann, dass alle Abkömmlinge aus einem bestimmten Laichklumpen sich anormal entwickeln. Selbstverständlich sind derartige Tiere nicht zu den Versuchen verwandt worden.

Bezüglich der mitgeteilten Abbildungen sei noch hervorgehoben, dass, wo nichts besonderes vermerkt ist, die Tiere 8-14 Tage unter dem Einflusse der betreffenden Substanz standen.

Die Versuche sind vor allen Dingen auch auf Axolotl, Tritonen und Wasserkäfer, ferner auf Copepoden ausgedehnt worden, und endlich sind mehrere Tausend von Wolfsmilchschwärmer-Raupen als Versuchstiere herangezogen worden. Die Absicht war, festzustellen, ob es möglich ist, die Raupen als solche in ihrem Wachstum zu beeinflussen. Ferner war die Möglichkeit gegeben, dass die Verpuppung früher oder später erfolgte, und endlich sollte geprüft werden, ob die Farbe der Raupen sich durch die Art der dargereichten Zusätze beeinflussen lässt. Bekanntlich zeigen die Wolfsmilchschwärmer-Raupen ein ausserordentlich buntes Bild. Es scheint; als ob durch Verabreichung von alkoholischèm Hefeextrakt die Zahl der dunkelgefärbten Raupen zunimmt, doch möchte ich einstweilen keine bestimmten Angaben machen. Auch hier gilt es, mehr Erfahrung zu sammeln. Die Raupen sind alle gezeichnet und gemalt worden, um so ein zuverlässiges Vergleichsmaterial für spätere Versuche zu haben.

Hinzufügen möchte ich noch, dass zahlreiche Versuche über den Einfluss der Färbung der Raupen auf diejenige der Schmetterlinge gemacht worden sind. Es gibt bei den Wolfsmilchschwärmer-Raupen ganz besondere Typen von Färbungen, zum Beispiel solche, deren Grundfarbe schwarz ist und die eine schwarze Rückenlinie besitzen. Andere haben eine rote Rückenlinje, wieder andere sind in der Hauptsache rötlich gefärbt. Es sind besonders die Seitenflecke rötlich. Wieder andere sind in der Hauptsache gelb bis gelbgrün gefärbt. Alle diese Färbungen kommen auch kombiniert vor. Wir haben eine grosse Zahl von solchen bestimmt gefärbten Raupen ausgesucht und dann verfolgt, was aus ihnen wurde. Es liess sich ein Zusammenhang der Färbung der Flägel der Schmetterlinge mit der Färbung der Raupen nicht feststellen.

Als besonderer Befund, der zufällig erhoben wurde, sei exwähnt, dass beim Auflegen von Schmetterlingsflügeln auf eine photographische Platte ohne jede Belichtung ein positives 
Bild entsteht ${ }^{1}$. Wir haben: diesen Versuch oft wiederholt; das Resultat war immer das gleiche, nur wurde ab und zu einmal ohne erkennbare Ursache auch ein negatives Bild erhalten. Auch die entschuppten Schmetterlingsflügel geben positive Bilder. Wir sind dabei, festzustellen, worauf diese Erseheinung beruht. In Frage kommt in erster Linie der Kalium- und der Purinbasengehalt der Flügel. Es soll auch géprüft werden, ob bereits die Raupen diese Einwirkung auf die photographische Platte zeigen. Die im vergangenen Jahr in dieser Richtung ausgeführten Versuche haben kein endgültiges Ergebnis gezeitigt.

Wir hahen vorläufig Versuche mit Purinbasen, Nukleinen, Nukleinsäuren und Nukleoproteiden ausgefïhrt und ierner geprüft, ob auch dann Bilder entstehen, wenn die Sshmetterlinge bezw. die genannten Substanzen der photographischen Platte nicht direkt au!liegen. Dass die letzteren bei direkter Berührung mit der Platte Wirkungen zeigen, ist nicht üterraschend. Es könnte sich hierbei um direkte chemische Einflüsse handeln. Die Seite 244 und 245 mitgeteilte Übersicht gibt einige der erhaltenen Resultate wieder. Die Versuche werden fortgesetzt.

Endlich interessierte die Frage, ob der Schmetterling als solcher irgendwie durch besonders gefütterte Raupen beeinflusst wird. Untersucht wurde die Grösse und die Farbe der Schmetterlinge. Ein einheitliches Resultat wurde leider nicht erhalten. Raupen, đie Hypophysensubstanzen erhalten hatten, ergaben zum Teil auffallend grosse Schmetterlinge. Ein Teil zeigte einen sehr grossen Körper, die Flügel dagegen waren ganz klein. Ferner zeigten auffallend viele Tiere rötlich gefärbte Vorderflügel. Tiere, die Schilddrüsensubstanz aufgenommen hatten, waren zum Teil anffallend klein und dabei wohl ausgebildet. Einzelne Tiere zeigten auf den Hinterflügeln ein sehr breites schwarzes Band, Zahlreiche Tiere zeigten un vollkommen entwickelte Flügel. Auffallend klein blieben die Tiere, die Nebennierensubstanz aufgenommen hatten. Auch hier waren zahlreiche missgestaltete Tiere vorhanden. Auffallend grosse Tiere wurden bei den Hodenversuchen beobachtet. Die Thymustiere zeigten besonders zahlreich auffallend blasse Vorderflügel. Mit Hefeextrakt - gewonnen durch Auskochen von Hefe mit Alkohol und Eindampfen der alkoholischen Lösung und Lösen des Rückstandes in Wasser - wurden im Durchschnitt auch recht grosse Tiere erhalten. Auffallend waren besonders die lebhaften Farben sowohl der Raupen: uils der Schmetterlinge. Die Strumaschmetterlinge waren im Durchschnitt klein. Das gleiche war bei den Sarkomtieren der Fall. Bei Verabreichung von abgebautem Carcinom wurden auffallend viele

1) Die gleiche Beobachtung hat kürzlich $G$. W olf in der Naturforschenden 'Gesellschaft in Basel mitgeteilt, wie ich einer Zeitungsnotiz entnehme. 
Weit. Studien üb. die von einz. Organen hervorgebrachten Substanzen. II. 243

missgestaltete Schmetteringe beobachtet. Mit Ovarium gefütterte Raupen exgaben Schmetterlinge, die besonders oft rötliche Vorderflügel besassen. In keinem einzigen Falle war jedoch das Resultat ein einheitliches. Es mag sein, dass die ausserordentlich schwierige Zufuhr der Substanzen an den unregelmässigen Resultaten schuld ist. Die Versuche wurden in der Weise rorgenommen, dass die zu prüfenden Substanzen in 1\%iger Lösung durch Zerstäuber auf die Wolfsmilchpflanzen geblasen wurden. Die Substanzen wurden während des Tages stündlich aufgehlasen. Die Wolfsmilchplanzen selbst wurden täglich ernénert. Es ist natürlich unter diesen Verhältnissen sehr schwer, für eine regelmässige nod in allen Fällen gleich gute Aufnahme der Substanzen Scrge zu tragen.

Endlich haben wir versucht, Einfluss auf die Entwjeklung von Ameisen zu gewinnen. Auch hier ist ein abschliessendes Urteil noch nicht möglich.

Ich möchte noch ausdrücklich betonen, dass der Plan für diese ganzen Versuche nicht nur von dem Gesichtspunkte aus aufgenommen. worden ist, eine Basis zum Studium der Wirkung der einzelnen Inkretstoffe zu gewinnen, sondern es sollte gleichzeitig die Möglichkeit geschaffen werden, die Inkrete mehrerer Organe in Mischungen anzuwenden. Eine geregelte Aufnahme der einzelnen Produkte war nur denkbar, indcm man sie einzeln löste und dann ihre Lösungen in bestimmten Anteilen mengte. Ich bin überzeugt, dass auf diesem Wege in Zukunft am besten die Zusammenhänge der einzelnen Organe sich latarstellen Iassein.

Jeider sind wir von der Reindarstellung der eixzelnen Inkretstoffe noch weit entfernt. Die ganzen Verslche sind dafurch ausserordentiich gehemmt, dass zurzeit die einzelnon Organe nur sehr schwer erhältlich sind. Dazu kommt noch der grosse Mangel an Chemikalien, der noch auf lange Zeit hinaus die wissenschaftiche Forschung sehr stark beschränken wird. Solange man nicht die einze!nen Substanzen als einboitlich definieren kam, kann man derartige Studien nur al; Vonliufer fur syatere exaktere betrachten. Sie stellen nur eine Stufe an einer Stufenleiter dar, die noch sehr viele Sprossen nach oben zeigt.

Besonders erwähnen wollen wir noch, dass sehr viele Untersuchungen ausgeführt worden sind, um bereits bekannte Substanzen auf ihre Wirkungen auf Wachstum und Entwicklung, speziell von Kaulcuappen, zu prüfen. Die Untersuchung der einzelnen Monoaminosäuren ergab, dass diesen keine spezifische Wirkung auf Wachstum und Entwicklung zukommt.

$\mathrm{Zu}$ den einzelnen mit Kaulquappen ausgeführten Versuchen ist ganz allgemein noch folgendes zu bemerken: 


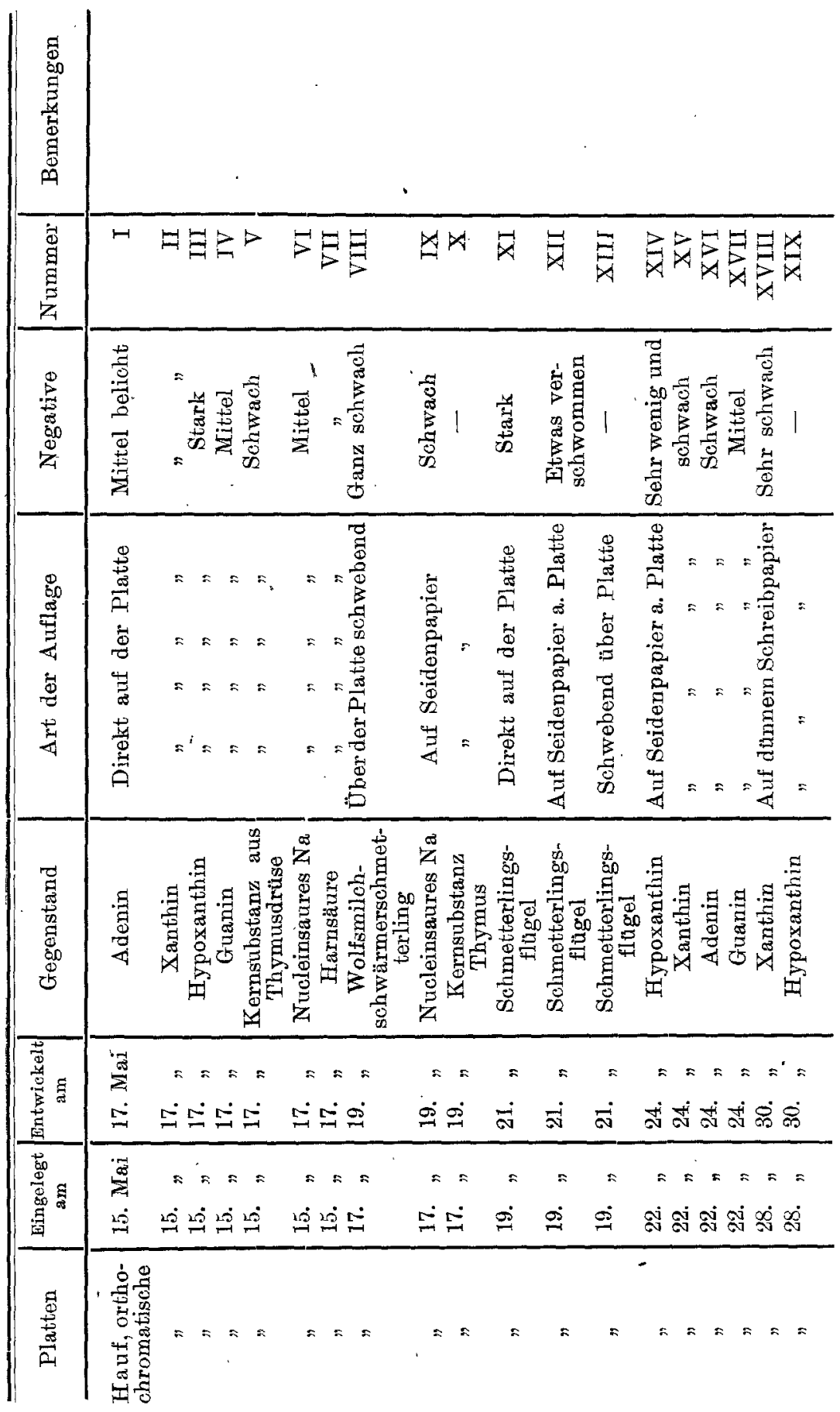


Weit. Studien üb. die von einz. Organen hervorgebrachten Substanzen. II. 245

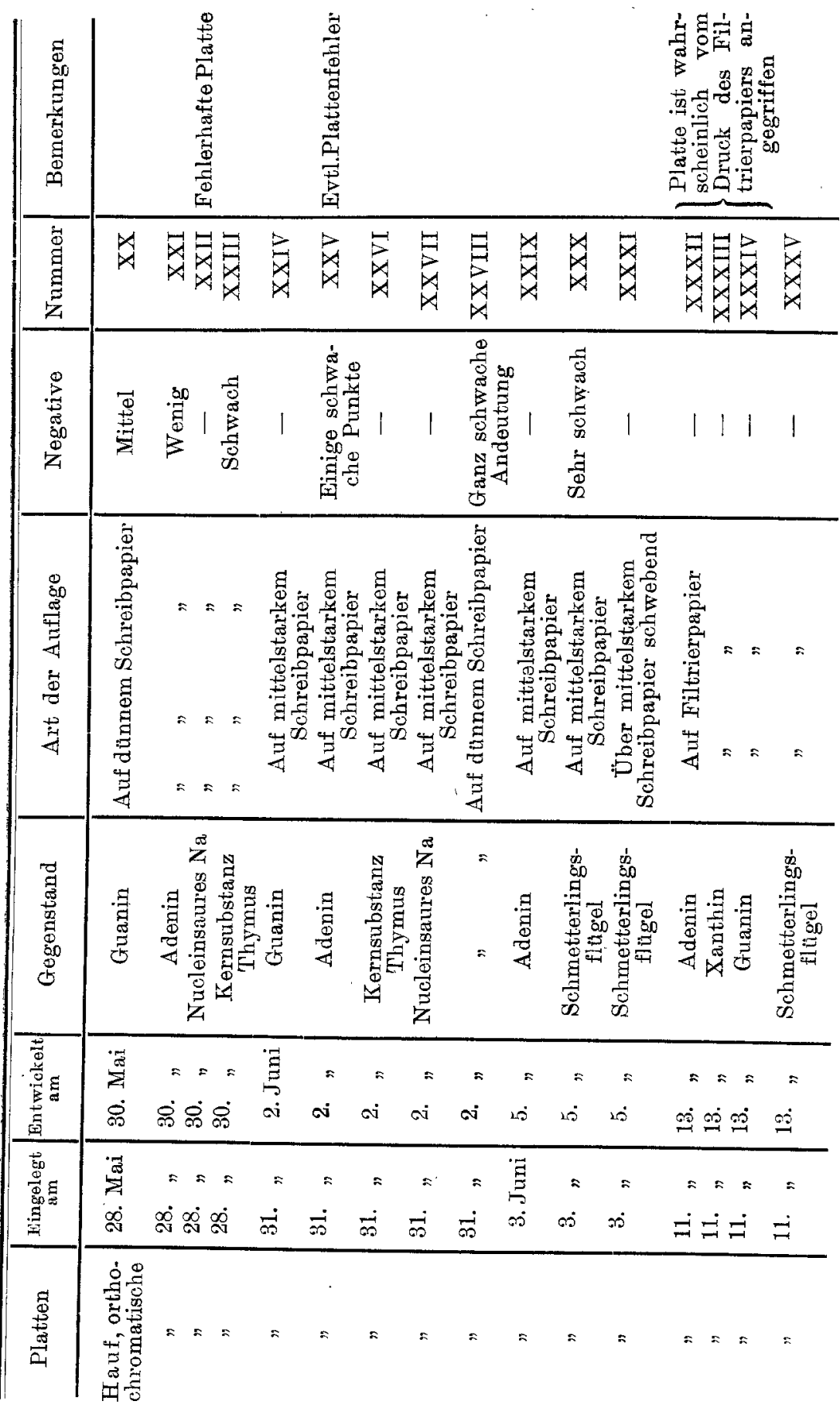


Die Versuchstiere wurden zu Gruppen vereinigt, und zwar bestanden diese aus einer grösseren Anzahl von Kontrolltieren, und dann folgten diejenigen Versuchstiere, die Zusätze erhielten. Jede Gruppe entstammte demselben Laich. Es handelte sich um Laich von Rana temporaria, Rana esculenta, Pelobates fuscus, Bombinator igneus, Bufo vulgaris, B. calamita. Sämtliche Versuchstiere befanden sich unter genau denselben Versuchsbedingungen. Sie wurden m Glasschalen (Durchmesser 14,3 cm, Höhe 4,6 cm) in $200 \mathrm{ccm}$ Wasser gehalten. Das Wasser wurde täglich oder alle zwei Tage عewechselt, um zu verhindern, dass Zersetzungsprozesse die zugefügten Stoffe beeinflussten. Ausserdem wollten wir stets über die Konzentration der angewandten Stoffe genau unterrichtet sein. Das Wasser wurde nie frisch aus der Leitung verwendet. Es wurde vielmehr schon am vorhergebenden Tage im gleichen Raume authewahrt. Diese Vorsichtsmassregei wurde angewandt, um Störungen im Woblbefinden der Tiere durch schroffe Temperaturänderungen zu rermeiden. Bei einigen Versuchen wurden Algen zugesetzt, bei anderen nicht. Es zeigte sich, dass die besten Resultate dann erhalten wurden, wenn der Zusatz von Algen oder anderer Nahrung unterblieb. Handelte es sich darum, die Tiere möglichst lange am Leben zu erhalten, dann mussten Algen dem Wasser zugefügt werden. Wir haben einzelne Versuchstiere 10 Monate beobachten können. Die Zahl der Versuche ist eine ausserordentlich grosse. Die Versuchstiere wurden täglich gemessen, und zwar wurde festgestellt: die Rumpflänge, die Schwanzlänge und die Rumpfbreite, wobei der breiteste Teil des Rumpfes gemessen wurde. Die gemachten Beobachtungen decken sich in vielen Teilen mit den bereits mitgeteilten Ergebnissen.

\section{Einfluss von Schilddrüsensubstanz anf Wachstum und Entwicklung.}

Zur Verwendung kam vollständig abgebaute Schilddrüsensubstanz - hydrolysiert durch Fermentwirkung oder durch verdünnte Schwefelsäure. Ferner wurde Schilddrüse nur schwach verdaut, und zwar durch Pankreassaft während 3 Tagen, und dann das gewonnene Produkt der Dialyse unterworfen und das Dialysat verwendet. Ferner sind Versuche ausgeführt worden, um den Einfluss von Schilddrüsen von BasedowKranken zu prüfen, und endlich sind auch Strumen dazu verwandt worden.Was die letzteren Versuche anbetrifft, so sind sie noch nicht zahlreich genug. Es scheint, als ob die Basedow-Schilddrüsen-Substanzen in vermehrtem Maasse auf die Kaulquappen einwirkten. Vor allen Dingen. starben auffallend viele Tiere. Bei Verwendung von Strumen waren die Resultate ungleichmässig, zum Teil blieb die die Metamorphose: 
Weit. Studien äb. die von einz. Organen hervorgebrachten Substanzen. II. 247

beschleunigende Wirkung ganz aus. Es entstanden Tiere, die mehr den Typus der Thymustiere zeigten. Bei manchen Fällen sahien eine Kombination von Wirkungen vorhanden zu sein. Die Tiere entwickelten sich schneller als die normalen, wurden aber gleichzeitig auch grösser. In diesen Fällen konnte man annehmen, dass neben der veränderten Schilddrüse noch normales Schilddrüsengewebe vorhanden gewesen und zur Verarbeitung gekommen ist. Vergleiche dazu die Abbildungen auf Tafel III-IV, 73-82.

Wir möchten auf diese Versuche keinen allzu grossen Wert legen, weil einerseits die Zahl der Versuche noch zu klein ist wnd andererseits die verwandten Organe histologisch nicht genügend geprüft worden sind. Vergleichbare Resultate werden sich erst dann erreichen lassen, wenn ganz gleichartige Gewebe zur Verwendung kommen. Vielleicht lässt sich auf dem betretenen Wege genauer feststellen, ob in den Fällen des Versagens der Schilddrüse im Organismus eine richtige Hyper- oder Hypofunktion vorliegt oder aber eine Dysfunktion.

Die mit den Schilddrüsensubstanzen erhaltenen Resultate waren bei allen verwendeten Batrachiem ganz gleichmässige. Wir haben jedoch den Eindruck gewonnen, dass es nicht unwesentlich ist, wann die Einwirkung auf die Kaulquappen stattfindet. In der ersten Zeit der Entwickliung scheint der Finfluss nicht so wesentlich zu sein als in späteren Entwicklungsstadien. Auch hierüber müssen noch mehr Erfahrungen gesammelt werden. Fs wäre von grösstem Interesse, ?u erfahren, ob zum Beispiel die Schilddrüsensubstanz ganz besonders dann ihre Wirkung zeigt, wenn es sich um die Entwicklung des Skelettes handelt. Die Haupterscheinung ist ohne Zweifel die starke Beeinflussung des Stoffumsatzes der Tiere. Es handelt sich bei der Einwirkung der Schilddrüsensubstanz nicht nur um eine stark beschleunigte Metamorphose, sondern es bleiben die Tiere zugleich klein. Sie nehmen an Körpergewicht sehr stark ab. Sobald man grössere Dosen von Schilddrüsenstoffen auf die Tiere einwirken lässt, erhält man ejgenartige Entwicklungsstörungen. Ihr Studium wird von grösstem Interesse sein. Es ist ganz gut denkbar, dass bestimmte Missbildungen, die sich auch beim Fötus der Säugetiere und des Menschen zeigen, auf ein Versagen entsprechender Drüsen mit Inkreten zurückzuführen sind. Die gemachten Beobachtungen seien an Hand einiger Protokolle belegt. Wir bemerken dazu, dass im ganzen 500 genaue Protokolle aufgenommen worden sind. Ferner geben die Abbildungen 1-30 auf Tafel III ein Bild der stattgefundenen Veränderungen. Man erkennt, dass der Hinterleib stark verschmälert ist. Das gleiche erkennt man bei den mit Schilddrüsendialysat bohandelten Tieren, wie die Abbildungen 52- 68 der Tafel IIT zeigen. Auf Tafel IV (84-88) sind Kaulquappen dargestellt, bei denen 
grössere Dosen von Schilddrüsensubstanz zu pathologisehen Veränderungen geführt haben. Bei den meisten dieser Fälle kam es nicht zur Entwicklung der vorderen Extremitäten, während die hinteren ausserordentlich rasch hervorsprossten. Die Tiere gingen meist sehr bald zugrunde. Gelang es, sie länger am Leben zu erhalten, dann erwiesen sie sich im Gegensatz zu den ,normalen“ Schilddrüsentieren als sehr träge und wenig beweglich.

Auf Tafel III sind in Abb. 31 a und $b$ zwei Axolotl abgebildet. Das Tier $a$ war der Schilddrüsensubstanzwirkung ausgesetzt, $b$ ist das gleichalterige normale Tier (Alter 4 Wochen). Vgl. auch die Tabellen: Gruppe I-IV S. 249-252.

\section{Einfluss von Thymussubstanz auf Wachstum und Entwicklung.}

Bei Verwendung von Thymussubstanz war der Erfolg immer derselbe. Die Tiere nahmen dauernd an Körpergrösse zu, ohne dass es zum Beginn bzw. zur Fortsetzung der Entwicklung $\mathrm{ka} \mathrm{m}$. Wir haben einzelne Tiere, die mit vollständig abgebauter Thymussubstanz gefüttert worden waren, bis 8 Monate am Leben erhalten. Die Tiere wuchsen dabei immer weiter, und es entstanden Riesenkaulquappen. Wir haben von Rana esculenta bedeutend grössere Kaulquappen erhalten, als sie bei Pelobates vorkommen. Einige Tiere zeigten insofern ein besonderes Verhalten, als der Rumpf sich mehr und mehr der Kugelform näherte. Die Tiere vermochten dann im Wasser das Gleichgewicht nicht mehr zu halten. Sie schwammen zum Teil in Seitenlage, zum Teil in Rückenlage. Bei diesen Tieren wurde ein auffallend grosses Herz gefunden. Es zeigte noch embryonale Züge. Bei vielen war auch der Rudersehwanz zu einer ganz aussergewöhnlichen Länge angewachsen.

Bei den Axoloteln war der Einfluss im grossen und ganzen nicht so ausgesprochen. Immerhin wurden auch hier ein paar ausgesprochene Fälle von verlangsamter Entwicklung beobachtet.

Auf Tafel IV sind eine Reihe von unter dem Einfluss von Thymussubstanz stehender Tiere abgebildet, und zwar sind alle Kaulquappen vom gleichen Alter, wie diejenigen, die auf Tafel III (Schilddrüsenwirkung) dargestellt sind.

Auch hier mögen einige Protokolle einen Einblick in die Ergebnisse der vorgenommenen Messungen der Körperlänge und -breite geben. (Tabellen Gruppe I-IV S. 253-256.)

\section{Einfluss von Hypophysensubstanz auf Wachstum und Entwicklnng.}

Die Ergebnisse mit Hypophysensubstanzen sind nicht einheitlich ausgefallen. Wir erhielten zum Teil eine sehr starke Zunahme des 
Weit. Studien üb. die von einz. Organen hervorgebrachten Substanzen. II. 249

\section{Gruppe.}

Gemessen am 1. Mai 1917, eine Woche nach Einwirkung der Inkretstoffe.

Alle Maasse sind in Zentimetern angegeben. Jeder Einzelversuch bestand aus 20 Tieren. Die einzelnen Werte sind Durchschnittswerte. Die Kaulquappen waren bei allen hier in Tabellenform mitgeteilten Versuchen 3-4 Wochen alt.

\begin{tabular}{|c|c|c|c|c|c|c|c|c|c|c|c|c|c|c|c|c|c|c|c|}
\hline \multicolumn{4}{|c|}{ Ohne Zusatz } & \multicolumn{4}{|c|}{$\begin{array}{l}\text { Schilddrüse } \\
\text { unabgebaut }\end{array}$} & \multicolumn{4}{|c|}{$\begin{array}{c}\text { Schilddrüse } \\
\text { durch Fermente } \\
\text { abgebaut }\end{array}$} & \multicolumn{4}{|c|}{$\begin{array}{c}\text { Schilddrüse } \\
\text { durch Säure } \\
\text { abgebaut }\end{array}$} & \multicolumn{4}{|c|}{$\begin{array}{l}\text { Basedow- } \\
\text { Schilddrüse } \\
\text { unabgebaut }\end{array}$} \\
\hline 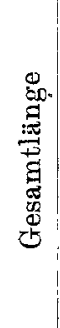 & 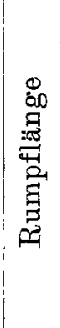 & 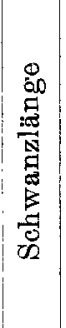 & 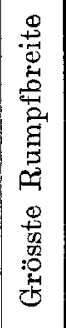 & 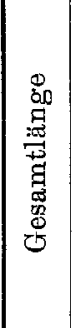 & 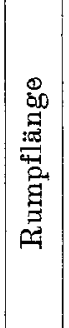 & 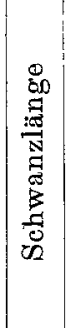 & 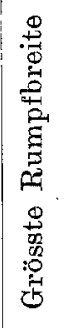 & 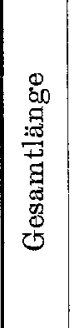 & 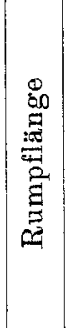 & 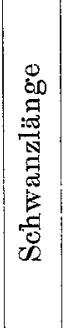 & 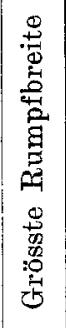 & 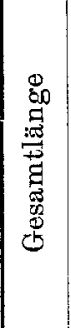 & 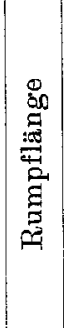 & 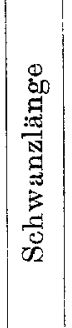 & 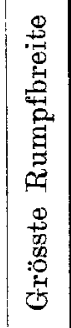 & 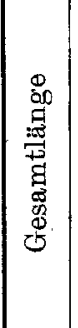 & 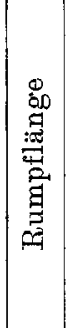 & 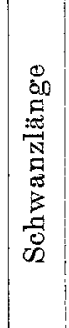 & 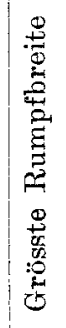 \\
\hline 1,80 & 0,54 & 1,26 & 0,60 & 1,75 & 0,50 & 1,25 & 0,48 & 1,80 & 58 & 1,220 & 0,52 & 1,5 & .054 & 1,00 & 0,40 & $\mid 1,20$ & 4 & 0, & 0,2 \\
\hline 1,79 & 180 & 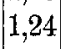 & 0,58 & 1,80 & 0,52 & 1,2 & 0,50 & 1,80 & & & & & & & 0,52 & & & & 0,32 \\
\hline 1,6 & 0 & & 0,61 & 1,69 & & & 0,51 & 1,69 & 2 & 1 , & 0,50 & 62 & & 1,30 & 0,48 & 1,45 & 0 & & 0,38 \\
\hline 1,75 & 0 & & 0,59 & 1,71 & 0,53 & & 0,52 & 1,75 & 0,61 & & 0,49 & 1,88 & & 1,41 & 0,49 & 1,43 & 0,41 & & 0,41 \\
\hline 1,90 & & & 0,58 & 1,58 & 0,56 & & 0,56 & 1,80 & $(0,44$ & 1,360 & 0,44 & 1,90 & & 1,30 & 0,32 & 1,44 & 4 & & 0,4 \\
\hline 2,10 & 0,61 & 1,49 & 0,58 & 1,62 & 0,61 & 0,0 & 0,58 & 1,69 & & 1,290 & 0,48 & 1,95 & & 1,62 & 0,40 & 1,42 & & & , \\
\hline 2,25 & 0,68 & & 0,59 & 1,75 & 0,60 & 1, & 0,40 & 1,69 & & $1,24 \mathrm{C}$ & 0,56 & 1,66 & & 1,12 & 0,39 & 1,40 & & & 0,38 \\
\hline 2,12 & 0,65 & & 0,61 & 1,80 & & & 0,45 & 1,65 & & 1,190 & 0,50 & 1,68 & & 8 & 0,45 & 1,58 & 5 & 8 & 0,41 \\
\hline 2,24 & 0,70 & 1,54 & 0,61 & 1,76 & & & 0,51 & 1,64 & 4 & 1,20 & 0,52 & 1,66 & 2 & 1,04 & 0,42 & 1,58 & 0,58 & 1,00 & 0,40 \\
\hline 2,30 & 0,69 & 1,61 & 0,60 & 1,82 & 0,60 & 1,2 & 0,42 & 1,68 & 0,49 & 1,19 & 0,51 & 1,68 & & 1,10 & 0,50 & 1,32 & 0,42 & 0,90 & 0,4 \\
\hline 1,86 & 0, & 1,0 & 0,58 & 1,81 & 0,62 & 1,19 & 0,39 & 1,75 & & 1,30 & 0,48 & 1,62 & & & 0,51 & 1,25 & 0,35 & & 0,48 \\
\hline 1,9 & 0,60 & 1,3 & 0,56 & 1,78 & 0,58 & 1,20 & 0,41 & 1,72 & & & 0,42 & & & & 0,52 & 1,45 & 0,39 & 1,06 & 0,49 \\
\hline 1,90 & 0,60 & 1,8 & 0,62 & 1,79 & & 1,29 & 0,45 & 1,69 & 0 & 1, & 0,40 & 1,66 & 6. & 1,00 & 0,38 & 1,46 & 0,46 & 1,00 & 0,51 \\
\hline 1,95 & 0,66 & 1,2 & 0,62 & 1,70 & 0 & 1,25 & 0,40 & 1,68 & 0,48 & 1,20 & 0,48 & 1,72 & & 1, & 0,32 & 1,58 & 0,48 & 1, & 0,5 \\
\hline 1,9 & 0,67 & 1,3 & 0,61 & 1,72 & 0,44 & 1,28 & 0,48 & 1,78 & & 1,38 & 0,51 & 1,80 & 0,55 & & 0,48 & 1,67 & & & 0 \\
\hline 1, & 0,68 & 1,2 & 0,61 & 1,68 & 0,50 & 1,18 & 0,42 & 1,85 & & 1,34 & 0,50 & 1,45 & & & 0,40 & 1,72 & 1 & 1 & 0,45 \\
\hline & & & 0,58 & 1,66 & & & 0,40 & 1,78 & 0 & 1,260 & 0,45 & 1,30 & 0 & 0 & 0,35 & 1,68 & 0,60 & 1,08 & 0,44 \\
\hline & 0,79 & 1,39 & 0,56 & 1,54 & & & 0,48 & 1,60 & 0,40 & 1,200 & 0,40 & 1,40 & 0,38 & 1,02 & 0,40 & 1,44 & 0,45 & 0,99 & 0,38 \\
\hline 2,10 & 0,75 & 1,35 & 0,61 & 1,45 & 0,45 & 1,000 & 0,49 & 1,66 & 0,42 & 1,240 & 0,48 & 1,48 & 0,42 & 1,06 & 0,44 & 1,20 & 0,40 & 0,80 & 0,8 \\
\hline 2 , & 0,70 & 1,31 & 0,54 & 1,60 & 0,49 & 1,11 & 0,36 & 1,65 & 0,45 & 1,200 & 0,51 & 1,45 & & & 0,45 & 1,00 & $0,35 \mid c$ & 0,65 & 0,29 \\
\hline & 0,70 & 1,30 & 0,58 & 1,75 & $0,50]$ & 1,25 & 0,38 & 1,68 & & & 0,52 & 1,45 & 0,39 & 1,06 & 0,46 & 1,45 & 0,35 & 1,10 & 0,45 \\
\hline & 0,72 & 1,28 & 0,61 & 1,42 & 0,42 & $1,00 \mid$ & 0,42 & 1,71 & 0,41 & 1,300 & 0,58 & 1,68 & 0,48 & 1,20 & 0,44 & 1,56 & 0,48 & 1,08 & 0,38 \\
\hline 2,10 & 0,71 & 1,39 & 0,58 & 1,90 & 0,60 & 1,30 & 0,44 & 1,75 & 0,45 & 1,300 & 0,48 & 1,62 & $0,40 \mid 1$ & 1,22 & 0,52 & 1,52 & 0,52 & 1,00 & 0,41 \\
\hline 2,38 & 0,80 & 1,58 & 0,61 & 1,32 & 0,40 & 0,92 & 0,30 & 1,45 & 0,35 & 1,10 & 0,42 & 1,44 & 0,401 & 1,04 & 0,51 & 1,58 & $|0,52|$ & 1,06 & 0,42 \\
\hline 2,14 & 0,72 & 1,42 & 0,62 & 1,65 & 0,48 & 1,17 & 0,54 & 1,68 & 0,48 & 1,200 & 0,48 & 1,40 & 0,401 & 1,00 & 0,48 & 1,44 & 0,42 & 1,02 & 0,3 \\
\hline & 0,80 & 1,30 & 0,66 & 1,71 & 0,581 & 1,13 & 0,52 & 1,65 & 0,45 & 1,200 & 0,49 & 1,59 & 0,511 & 1,08 & 0,51 & 1,68 & 0,56 & 1,12 & 0,41 \\
\hline 2,16 & 0,75 & 1,41 & 0,59 & 1,82 & 0,60 & 1,22 & 0,58 & 1,70 & 0,60 & 1,100 & 0,51 & 1,48 & 0,42 & 1,06 & 0,45 & 1,250 & 0,40 & 0,850 & 0,44 \\
\hline 2,19 & 0,72 & 1,47 & 0,58 & 1,70 & 0,45 & & 0,52 & 1,66 & 0,46 & 1,20 & 0,52 & 1,52 & \begin{tabular}{ll|l}
0,52 & 1
\end{tabular} & 1,00 & 0,38 & 1,18 & 0,380 & 0,800 & 0,42 \\
\hline
\end{tabular}


II. Gruppe.

Gemessen an 10. Mai 1918, 10 Tage nach der Einwirkung der Inkretstoife.

Rana esculenta.

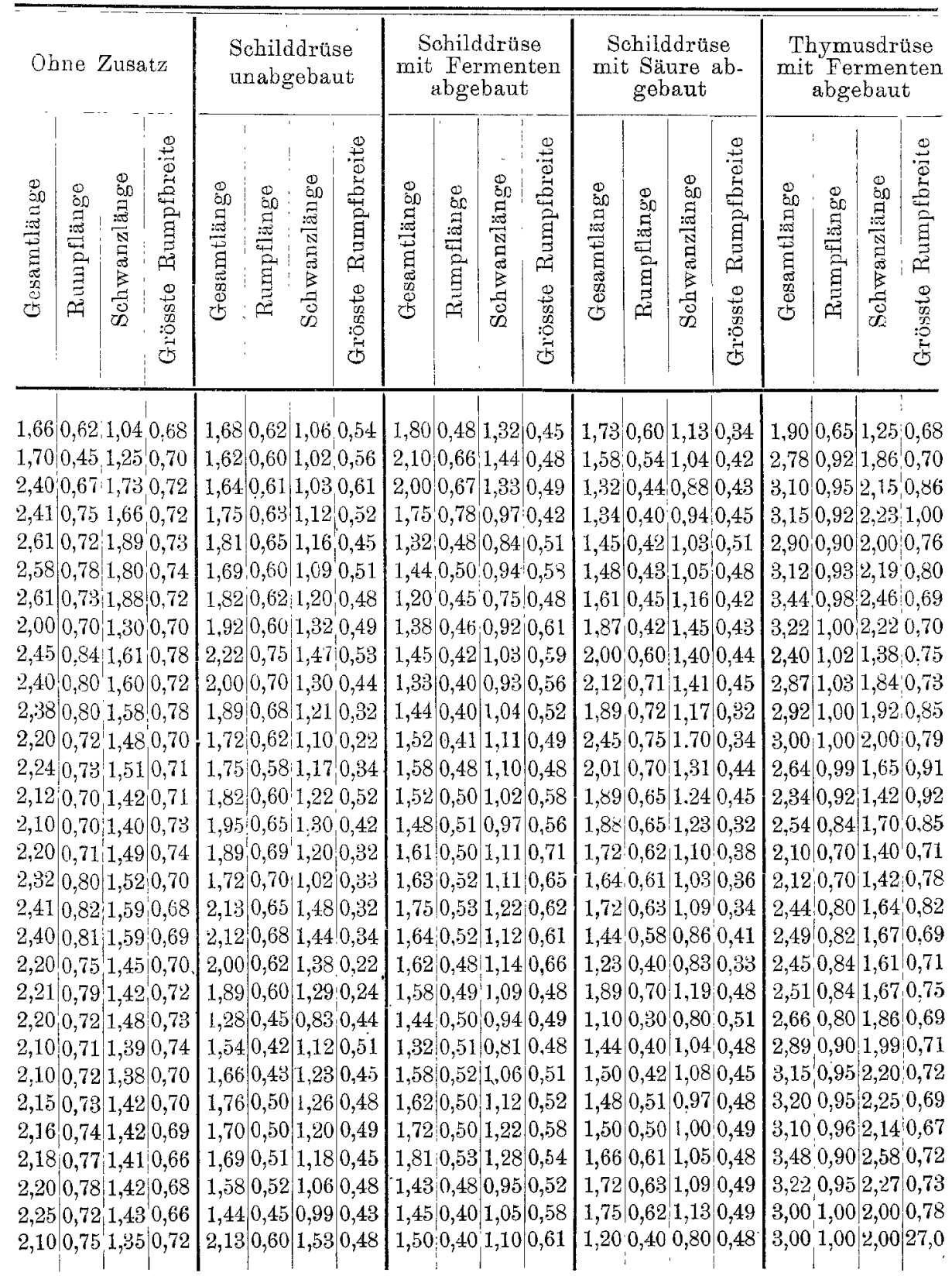


Weit. Studien üb. die von einz. Organen hervorgebrachten Substanzen. II. 251 IIT. Gruppe.

Gemessen am 16. April 1917, 20 Tage nach der Einwirkung der Inkretstoffe. Rana temporaria.

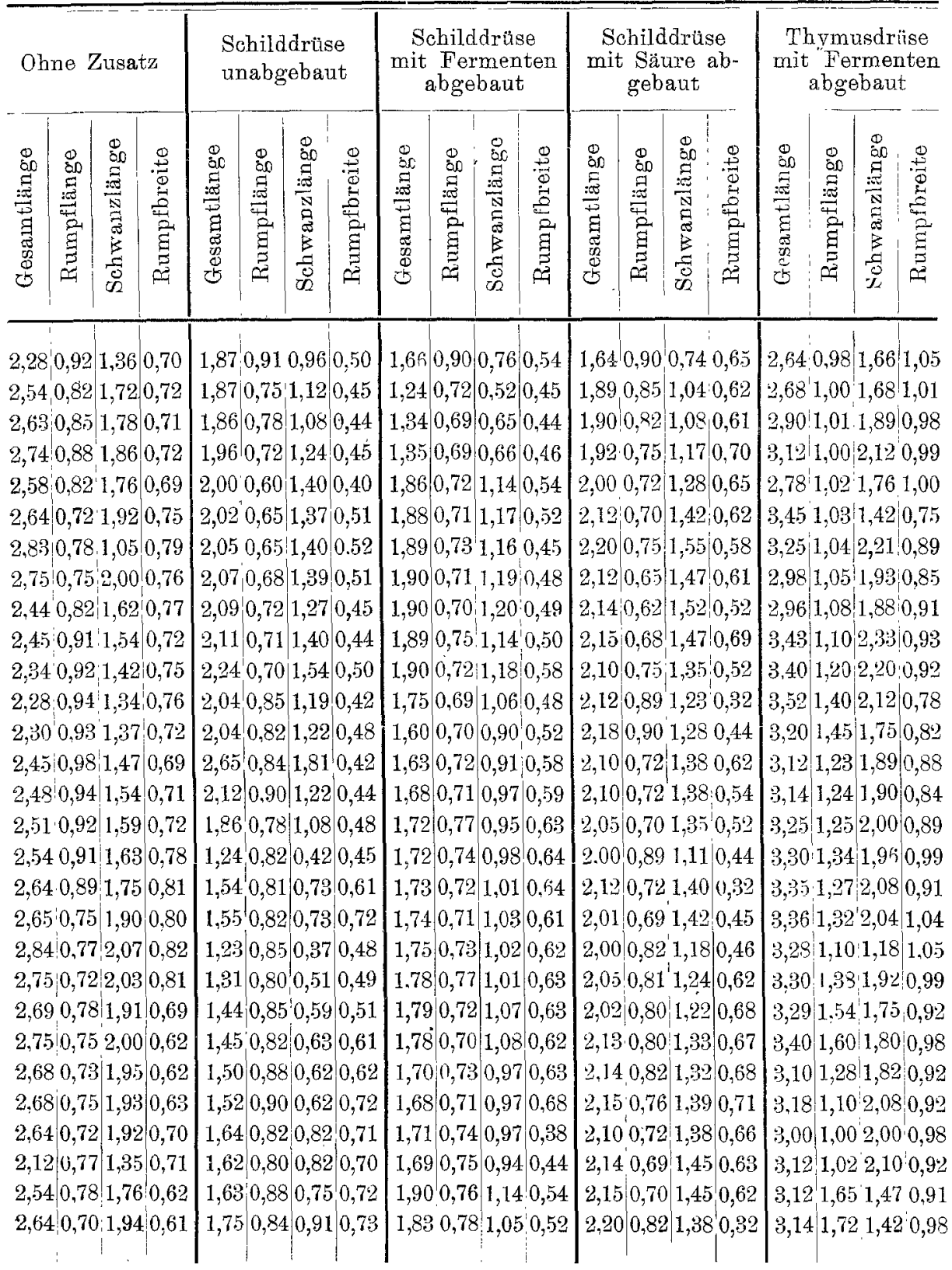


IV. Gruppe.

Gemessen am 15. Mai 1918, 14 Tage nach der Einwirkung der Inkretstoffe. Bufo vulgaris.

\begin{tabular}{|c|c|c|c|c|c|c|c|c|c|c|c|c|c|c|c|c|c|c|c|}
\hline \multicolumn{4}{|c|}{ Ohne Zusatz } & \multicolumn{4}{|c|}{$\begin{array}{l}\text { Schilddrüse } \\
\text { unabgebaut }\end{array}$} & \multicolumn{4}{|c|}{$\begin{array}{l}\text { Schilddrüse } \\
\text { mit Fermenten } \\
\text { abgebaut }\end{array}$} & \multicolumn{4}{|c|}{$\begin{array}{c}\text { Schilddrusse } \\
\text { mit Säure ab- } \\
\text { gebaut }\end{array}$} & \multicolumn{4}{|c|}{$\begin{array}{l}\text { Thymusdrüse } \\
\text { mit Fermenten } \\
\text { abgebaut }\end{array}$} \\
\hline 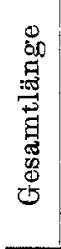 & 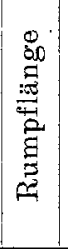 & 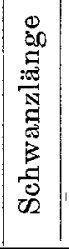 & 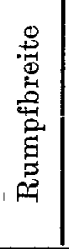 & 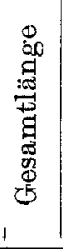 & 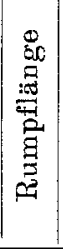 & 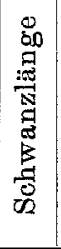 & 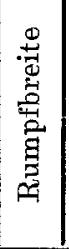 & 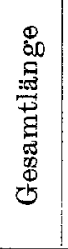 & 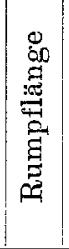 & 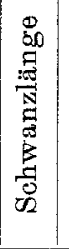 & 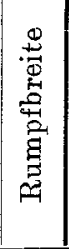 & 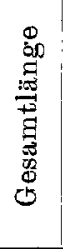 & 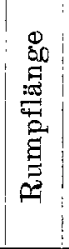 & 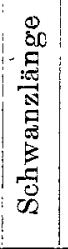 & 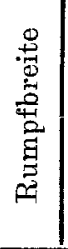 & 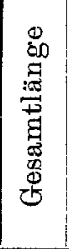 & 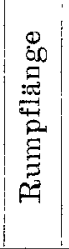 & 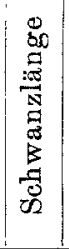 & 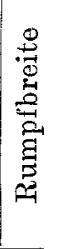 \\
\hline & &, 3 &, 52 & 1, & jo & & 0,38 & $\mid 1,46$ & 0,76 & & 38 & & \% & & 0,66 & & & & 100 \\
\hline & . & & 0,61 & 1,0 & & & 0,42 & & & & 44 & & & & 0,54 & & & &, 0 \\
\hline &, 65 & 0,9 & 0,52 & 1,21 & 0, & & 0,42 & 1,34 & 0,62 & 0 & 0,56 & 1, & $a-1$ & & 0,44 & 2 & 7 & & 0,70 \\
\hline & 3 & 0,9 & 0,48 & 1,11 & 0 & & 0,48 & 1,42 & 0,67 & & 0,48 & 1 & 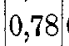 & & 0,45 &, 15 & $0-1$ & & 0,7 \\
\hline & 2 & 0, & 0,46 & 1,1 & & & 0,42 & 1,45 & 0,72 & & 51 & 1,50 & & & 0,40 & 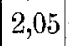 & & & 0,70 \\
\hline 1, & 2 & 0,8 & 0,48 & 1. & & & 0,43 & 1,46 & & & 61 & & & 0 & 0,51 & 92 & 1 & & 0,82 \\
\hline & & & 0,42 & & & & 0,50 & 1,48 & & & 0,71 & & & ( & 0,58 & & & &, 4 \\
\hline 1, & 0 & 0,8 & 0,45 & 1, & 0 & & 0,51 & 1,50 & 0 & & 0,25 & & & 0 & 62 & t & 14 & &, 8 \\
\hline & & 1, & 0,48 & 1,22 & & & 0,48 & 1,44 & & & 0,48 & & & & 0,58 & & & & 0,70 \\
\hline 1,50 & 0,52 & 0,9 & 0,42 & 1,00 & 0 & & 0,42 & $1,62 \mid$ & 0 & 0 & 0,44 & 1,42 & 2 & 0 & 0,59 & 9 & 1 & & 0,72 \\
\hline & & & $0 ; 43$ & & & & 0,48 & & & & & & & & 0,62 & & & & \\
\hline 1, & 0,51 & 1,00 & 0,42 & 1,02 & 0,46 & 0,56 & 0,41 & 1,66 & 0 & & 0,46 & 1,52 & & 0 & 0,68 & 1,94 & 1 & & 0,75 \\
\hline 1. & & & 0,41 & & & & 0,48 & & & & & & & & 0,62 & & & & 0,60 \\
\hline 1, & 0 & 0, & 0,38 & $1,1 \%$ & & 0 & $0,50 \mid$ & 1,44 & 0 & 0 & 0,48 & 1,4 & & 10 & 0,68 & 2,10 & & & 0,66 \\
\hline 1,54 & & & 0,39 & 1,00 & & & 0,22 & 1 & & & 18 & & & & 0,42 & & 68 & & \\
\hline 1,6 & 0, & 1, & 0,48 & 1,02 & & 0,52 & 0,24 & 1,32 & 0 & 0 & 0,45 & 1,22 & 2 & 0,60 & 0,40 & 12 & 0,65 & 1 & 0,66 \\
\hline 1,72 & 0,62 & 1 , & 0,42 & 1,23 & 0 & & 0,32 & 1,22 & 0 & 0 & 0,46 & 1,44 & 1 & 0 & 0,43 & 20 & 0,65 & 1 & 0,68 \\
\hline 18 & 8 & 1,12 & 0,48 & 008 & 0, & & 0,44 & 1, & 0 , & & 0.72 & & & & 0,48 &, 15 & 5 & & 0,62 \\
\hline 1,72 & 0 & 1,0 & 0,50 & 0,75 & 0 & 0 & 0,28 & 1,36 & 0,60 & & 0,61 & 1,50 & 0,62 & 0 & 0,58 &, 44 & c & & 0,75 \\
\hline & 0,71 & 1,1 & 0,52 & 0.9 & 0, & & 0,32 & 1,41 & 0,70 & & 0,61 & 1,52 & 0,68 & 0,84 & 0,52 & & 0. & & 0,7 \\
\hline 1,8 & 0,72 & 1,0 & 0,52 & 1,01 & 0 & 0,50 & 0,21 & 120 & 0 & 0 , & 0,46 & 1,54 & 0,70 & 0,84 & 0,60 & 1,60 & 0,52 & 1 & 0,48 \\
\hline & 0,60 & 0,84 & 0,49 & 1,02 & & & 0,31 & 1,25 & 0,60 & 0 & 0,32 & 18 & & 0,90 & 0,60 & 1,78 & 1 & 1 & 0,42 \\
\hline 1,4 & 0,62 & 0,8 & $0,4 ૪$ & 1,08 & 0,52 & 0,56 & 0,38 & 1,26 & 0 & 0 & 0,44 & 1,24 & 72 & 0,52 & 0,62 & 1,85 & 0,58 & 1,27 & 0,66 \\
\hline 1,5 & 0,61 & 0,89 & 0,49 & 109 & & & 0,4 & 1,28 & 0,72 & 0. & 0,45 & 1,10 & 0,70 & 0,40 & 0,61 & 1.92 & 0,65 & 1 & 0,68 \\
\hline 1,5 & 0,62 & 0.88 & 0,48 & 1,05 & 0 & 0,50 & 11,38 & 1,31 & 0,72 & & 0,32 & & & & 0,41 & 1,80 & 0,60 & & 0,62 \\
\hline 1,5 & 0,63 & $3 \mid 0,87$ & 0,49 & 1,25 & 0.65 & 0,60 & 0,41 & 1,29 & 0,71 & 0,58 & 0,31 & 1,13 & 0,54 & $|0,59|$ & 0,38 & 1,60 & 0,52 & 1 & 0,58 \\
\hline 1,4 & 50 & 0,81 & 0,48 & 1,34 & & 0,63 & 0,51 & 1,44 & 0,80 & 0,64 & 0,44 & 1,14 & 0,51 & $0,63 \mid$ & 0,32 & 1,58 & 0,51 & 7 & 0,52 \\
\hline 1,42 & 0,62 & 0,80 & 0,45 & 1,02 & 0,52 & 0,50 & 0,42 & 1,00 & 0,52 & 0,48 & 0,51 & 1,15 & 0,50 & 0,65 & 0,31 & 1,60 & 0,50 & 1 & 0,51 \\
\hline 1,44 & 40,63 & \begin{tabular}{l|l}
3 & 0,81
\end{tabular} & 0,46 & 1,04 & 0,54 & 0,50 & 0,34 & $1,2 ?$ & 0,60 & 0,63 & 0,51 & 1,18 & 0,50 & 0,68 & 0,30 & 1,77 & 0,58 & & 0,52 \\
\hline 1,4 & & 10,82 & 0,48 & 1,05 & 0,54 & 0,51 & 0,23 & 1,24 & 40,76 & 0,58 & 0,52 & 1,22 & 0,50 & 0,72 & 0,38 & 1,79 & 0,59 & 1,2 & 0,58 \\
\hline 1,42 & & & $0,+0$ & 1,00 & & & TU & 1,38 & 0,74 & {$[0,04$} & $: 0,53$ & 1,23 & 0,01 & ? & {$[0,0 y$} & 1,85 & $(0,00$ & $1,-20$ & 0,62 \\
\hline
\end{tabular}


Weit. Studien üb. die von einz. Organen hervorgebrachten Substanzen. II. 253

\section{Gruppe.}

Gemessen am 1. Mai 1917, eine Woche nach der Einwirkung der Inkretstoffe. Rana fusca.

\begin{tabular}{|c|c|c|c|c|c|c|c|c|c|c|c|c|c|c|c|}
\hline \multicolumn{4}{|c|}{ Ohne Zusatz } & \multicolumn{4}{|c|}{$\begin{array}{c}\text { Thymusdrüse } \\
\text { unabgebaut }\end{array}$} & \multicolumn{4}{|c|}{$\begin{array}{l}\text { Thymusdrüse } \\
\text { durch Fermente } \\
\text { abgebaut }\end{array}$} & \multicolumn{4}{|c|}{$\begin{array}{c}\text { Thymusdrüse } \\
\text { durch Säure } \\
\text { abgebaut }\end{array}$} \\
\hline 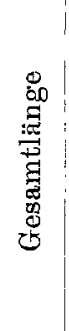 & 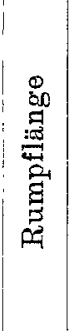 & 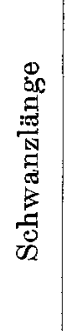 & 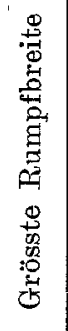 & 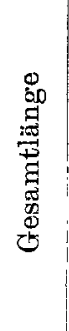 & 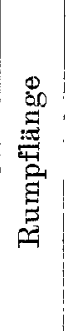 & 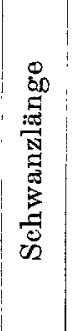 & 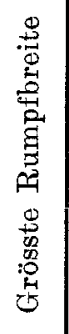 & 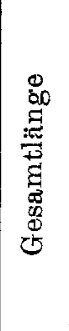 & 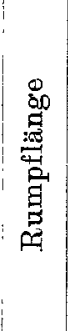 & 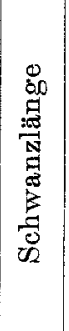 & 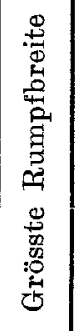 & 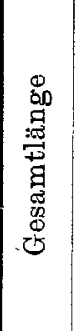 & 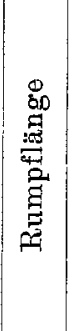 & 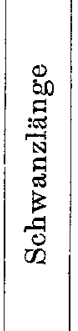 & 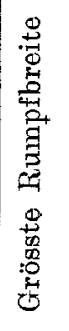 \\
\hline 75 & 0,56 & 1,19 & 0,62 & 2,00 & 0,79 & 1,21 & 1,00 & 1,95 & 0,65 & 1,30 & 0,85 & 1,99 & 0,79 & 1,20 & 0,78 \\
\hline 1,80 & 0,62 & 1,08 & 0,60 & 1,86 & 0,66 & 1,20 & 0,96 & 2,15 & 0,70 & 1,45 & 0,86 & 2,54 & 0,80 & 1,74 & 0,76 \\
\hline 1,98 & 0,75 & 1,23 & 0,59 & 1,95 & 0,66 & 1,29 & 0,90 & 2,38 & 0,78 & 1,60 & 0,91 & 2,34 & 0,74 & 1,60 & 0,77 \\
\hline 1,95 & 0,65 & 1,33 & 0,63 & 2,25 & 0,75 & 1,50 & 0,99 & 2,45 & 0,80 & 1,65 & 0,99 & 2,32 & 0,75 & 1,57 & 0,80 . \\
\hline 1,76 & 0,76 & 1,00 & 0,58 & 2,30 & 0,60 & 1,70 & 0,68 & 2,51 & 0,85 & 1,66 & 0,75 & 2,10 & 0,70 & 1,40 & 0,75 \\
\hline 1,97 & 0,75 & 1,22 & 0,61 & 2,40 & 0,80 & 1,60 & 0,89 & 2,11 & 0,70 & 1,41 & 0,68 & 2,37 & 0,69 & 1,68 & 0,69 \\
\hline 1,85 & 0,58 & 1,27 & 0,65 & 2,86 & 1,16 & 1,70 & 0,69 & 1,89 & 0,89 & 1,00 & 0,78 & 2,41 & 0,66 & 1,75 & 0,75 \\
\hline 1,90 & 0,65 & 1,25 & 0,62 & 2,66 & 1,06 & 1,60 & 0,71 & 1,90 & 0,65 & 1,25 & 0,72 & 2,32 & 0,72 & 1,60 & 0,85 \\
\hline 1,85 & 0,65 & 1,20 & 0,61 & & & & & 88 & & & 0,85 & & 0,68 & 1,50 & 0,90 \\
\hline 1,82 & 0,60 & 1,22 & 0,63 & 2,20 & 0,89 & 1,31 & 0,70 & 1,95 & 0,65 & 1,30 & 0,92 & 2,00 & 0,60 & 1,40 & 0,77 \\
\hline 1,88 & 0,62 & 1,26 & 0,65 & 2,44 & 0,90 & 1,54 & 0,69 & 2,34 & 0,74 & 1,60 & 0,82 & 1,80 & 0,55 & $1,2.5$ & 0,72 \\
\hline 1,90 & 0,60 & 1,30 & 0,66 & 2,89 & 1,04 & 1,85 & 0,71 & 2,25 & 0,70 & 1,55 & 0,69 & 1,65 & 0,45 & 1,20 & 0,75 \\
\hline 1,78 & 0,50 & 1,28 & 0,58 & 2,14 & 0,84 & 1,30 & 0,89 & 2,60 & 0,80 & 1,80 & 0,72 & 1,82 & 0,50 & 1,32 & 0,77 \\
\hline 1,80 & 0,62 & 1,18 & 0,61 & 1,85 & 0,85 & 1,00 & 1,00 & 2,75 & 1,00 & 1,75 & 0,78 & 1,92 & 0,62 & 1,30 & 0,80 \\
\hline 1,82 & 0,62 & 1,20 & 0,66 & 1,98 & 0,80 & 1,18 & 0,75 & 2,81 & 1,10 & 1,71 & 0,88 & 2,00 & 0,80 & 1,20 & 0,75 \\
\hline 1,75 & 0,65 & 1,10 & 0,62 & 2,44 & 0,90 & 1,54 & 0,89 & 2,79 & 1,00 & 1,79 & 0,82 & 3,12 & 1,38 & 1,74 & 0,89 \\
\hline 1,60 & 0,60 & 1,00 & 0,56 & 2,76 & 1,04 & 1,72 & 0,80 & 2,75 & 1,10 & 1,65 & 0,90 & 2,68 & 0,90 & 1,78 & 0,90 \\
\hline 1,89 & 0,62 & 1,27 & 0,58 & 2,75 & 1,00 & 1,75 & 0,81 & 2,22 & 0,72 & 1,50 & 0,89 & 2,71 & 0,90 & 1,81 & 0,90 \\
\hline 1,95 & 0,68 & 1,27 & 0,61 & 2,70 & 1,00 & 1,70 & 0,82 & 2,10 & 0,62 & 1,48 & 0,82 & 2,75 & 0,92 & 1,83 & 0,90 \\
\hline 2,55 & 0,65 & 1,90 & 0,71 & 2,45 & 0,90 & 1,55 & 0,85 & 1,90 & 0,60 & 1,30 & 0,82 & 2,81 & 0,90 & 1,91 & 0,92 \\
\hline 2,20 & 0,68 & 1,52 & 0,60 & 2,44 & 0,89 & 1,55 & 0,79 & 1,89 & 0,60 & 1,29 & 0,72 & 2,91 & 1,00 & 1,91 & 0,91 \\
\hline 2,15 & 0,70 & 1,45 & 0,56 & $2,48^{\circ}$ & 0,90 & 1,58 & 0,74 & 1,77 & 0,70 & 1,07 & 0,65 & 2,42 & 0,90 & 1,52 & 0,75 \\
\hline 2,00 & 0,65 & $1,35$. & 0,54 & 2,50 & 0,90 & 1,60 & 0,75 & 1,98 & 0,88 & 1,10 & 0,71 & 2,32 & 0,70 & 1,62 & 0,69 \\
\hline 1,98 & 0,60 & 1,38 & 0,61 & 2,61 & 0,86 & 1,75 & 0,65 & 2,24 & 0,75 & 1,49 & 0,58 & 2,00 & 0,60 & 1,40 & 0,77 \\
\hline 1,90 & 0,62 & 1,28 & 0,68 & 2,20 & 0,60 & 1,60 & 0,85 & 2,44 & 0,80 & 1,64 & 0,61 & 2,12 & 0,72 & 1,40 & 0,69 \\
\hline 1,95 & 0,61 & 1,34 & 0,62 & 2,00 & $0,61\}$ & 1,39 & 0,69 & 2,20 & 0,73 & 1,47 & 0,72 & 2,10 & 0,70 & 1,40 & 0,66 \\
\hline 1,98 & 0,66 & 1,32 & 0,60 & 1,98 & 0,60 & 1,38 & 0,71 & 2,12 & 0,75 & 1,87 & 0,78 & 2,00 & 0,70 & 1,30 & 0,60 \\
\hline
\end{tabular}


II. Gruppe.

Gemessen am 10. Mai 1918, 10 Tage nach der Einwirkung der Inkretstoffe. Rana esculenta.

\begin{tabular}{|c|c|c|c|c|c|c|c|c|c|c|c|c|c|c|c|c|c|c|c|}
\hline \multicolumn{4}{|c|}{ Ohne Zusatz } & \multicolumn{4}{|c|}{$\begin{array}{l}\text { Thymusdrüse } \\
\text { unabgebaut }\end{array}$} & \multicolumn{4}{|c|}{$\begin{array}{c}\text { Thymusdrüse, } \\
\text { mit Fermenten } \\
\text { abgebaut }\end{array}$} & \multicolumn{4}{|c|}{$\begin{array}{l}\text { Thymusdrüse } \\
\text { mit Säure ab- } \\
\text { gebaut }\end{array}$} & \multicolumn{4}{|c|}{$\begin{array}{l}\text { Schilddrüse } \\
\text { mit Fermenten } \\
\text { abgebaut }\end{array}$} \\
\hline 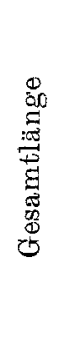 & 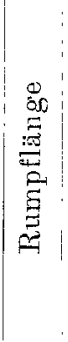 & 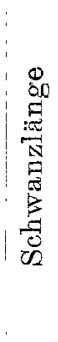 & 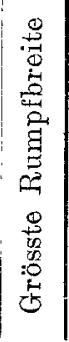 & 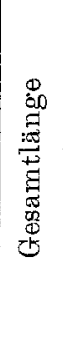 & 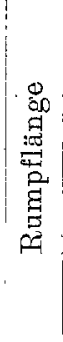 & 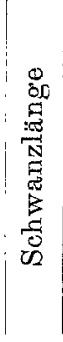 & 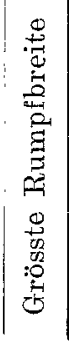 & 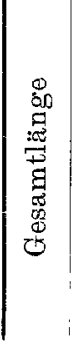 & 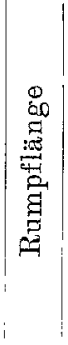 & 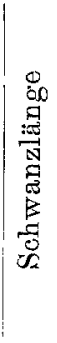 & 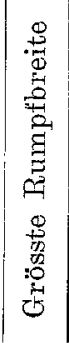 & 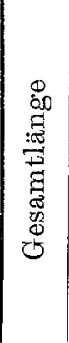 & 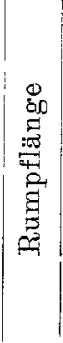 & 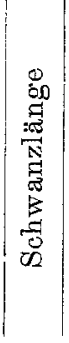 & 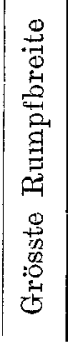 & 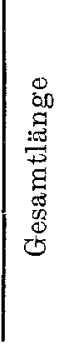 & 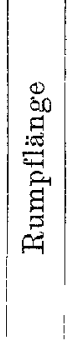 & 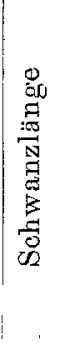 & 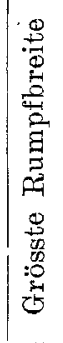 \\
\hline \multicolumn{4}{|c|}{ 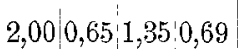 } & \multicolumn{4}{|c|}{$2,34|0,72 \quad 1,62| 0,75$} & \multicolumn{2}{|c|}{2,$46 ; 0,81$} & & 0,68 & \multicolumn{2}{|c|}{$3,101,00$} & \multicolumn{2}{|c|}{$2,10 \mid 0,82$} & \multicolumn{4}{|c|}{\begin{tabular}{l|l|l|l|l}
1,56 & 0,54 & 1,02 & 0,62
\end{tabular}} \\
\hline & & 1,62 & 0,68 & \multirow{2}{*}{\multicolumn{4}{|c|}{\begin{tabular}{|l|l|l|}
2,44 & $0,811,63,0,81$ \\
2,12 & $0,701,42,0,92$ \\
\end{tabular}}} & \multirow{2}{*}{\multicolumn{2}{|c|}{2,45}} & & 0,62 & 3,40 & & & 0,84 & \multicolumn{4}{|c|}{\begin{tabular}{ll|l|l|l|l|l|l|l|}
1,34 & 0,48 & 0,86 & 0,42
\end{tabular}} \\
\hline & & & & & & & & & & & 0,71 & 3 & & & 85 & \multicolumn{4}{|c|}{\begin{tabular}{l|l|l|l|l|l|l|}
1,56 & 0,51 & 1,05 & 0,44
\end{tabular}} \\
\hline & & & 72 & \multicolumn{4}{|c|}{\begin{tabular}{|l|l|l|l|l|l|l|l|l|l|l|l|l|}
2,12 & 0,70 & 1,42 & 0,92 \\
2,38 & 0,82 & 1,56 & 0,82
\end{tabular}} & 2,63 & & & 0,72 & & & & 0,80 & \multicolumn{4}{|c|}{\begin{tabular}{|l|l|l|l|}
, 200 & 0,31 & 1,00 & 0,44 \\
1,24 & 0,41 & 0,83 & 0,32
\end{tabular}} \\
\hline & & & 74 & \multicolumn{4}{|c|}{$2,480,84 \quad 1,64 \mid 0,83$} & 2,45 & 0 & & 75 & 2 & & & 0,72 & & & & 0,21 \\
\hline & & & 72 & 2,86 & 0,92 & 1,94 & 0,66 & 2,46 & & & 79 & & & & 0,70 & 4 & & &, 3 \\
\hline & & & 0,73 & 3, & & & & 2,39 & 0,82 & & 3 & & & & 77 & 1,76 & & & \\
\hline & & & 0,72 & & & & & 2,48 & & & & & & & 0 & 75 & & & \\
\hline & & &, 77 & 2 & & & & 2,43 & & & 30 & 2 & & & 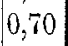 & & & & 32 \\
\hline & & &, 72 & & & & & 2,65 & & & 0,82 & & & & 1 & & & & 49 \\
\hline & & &, 65 & 3 & & & & 2,00 & & & 92 & 2, & & & 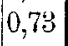 & & & & \\
\hline & & & 0,63 & 3 & & & & 2, & & & 95 & & & & & 0 & & & \\
\hline & & & 64 & 3,45 & & & & 2 & & & 72 & & & & & 52 & & & 2 \\
\hline & & & 0,64 & 3,20 & & & & 3 & & & 75 & 2 & & & 71 & & & & 283 \\
\hline & & & 0,62 & & & & & 2, & & & 0,71 & & & & 8?) & & & & 32 \\
\hline & & & 0,66 & & & & 1,00 & 2 & & & 0,69 & & & & 20 & & & & 34 \\
\hline & & & & & & & 0,92 & & & & 0 & & & & 8 & 1,72 & & & \\
\hline & & 1 & 62 & 2,61 & & & 92 & & & & 0 & & & & 70 & 1,54 & & & \\
\hline & & & 0,63 & & & $1^{2}$ & & 2,25 & & & 0,69 & & & & & & & & \\
\hline & & 1 & 0,48 & & 0 & & 0,90 & 2,14 & & 1 , & 0,68 & & & & 0,62 & 1,51 & 0 & 1 & 0,24 \\
\hline & & & 0,48 & & & & 0,92 & & & & 0,64 & 2,10 & & 1 & 0,61 & 1,54 & & & 0,48 \\
\hline & & & 0,53 & & & & 0,86 & & & & 0,75 & 2,38 & & & 0,78 & 1,48 & & & 0,42 \\
\hline $\overrightarrow{2}$ & 0 & $\overrightarrow{1}$ & 0,68 & 2,75 & & 1 & 0,84 & 2,29 & & 1 & 0,72 & 2 & 0 & & 0,70 & 1,24 & 6 & & 0,86 \\
\hline 2,30 & 0 & 1 , & 0,65 &, 89 & & & 0,92 & 2,12 & & & 0,70 & & & & 0,68 & 1,54 & & & 0 \\
\hline 244 & & 1, & 0,59 & 2,90 & 0 & 1 & 0,84 & 3,12 & 10 & 2,14 & 0,79 & 1,98 & & & 0,72 & 1,63 & 3 & & 0,48 \\
\hline & & & 0,61 & 1036 & & 1,61 & & 3,24 & 0,99 & 2,25 & 0,80 & 2,18 & 0,72 & 1,46 & 0,86 & 1,52 & 0,50 & 1 & 0,46 \\
\hline & & & 0,63 & & & & 0,81 & 3,00 & & 2,02 & 0,89 & 3,12 & 0,98 & 2,14 & 0,79 & 1,63 & 0,51 & & 0,47 \\
\hline & & & 0,65 & & 0 & & 0,82 & 3,18 & 0 & & 0,88 & 2,98 & 1,00 & 1,98 & 0,80 & 1,64 & 0,53 & & 0,46 \\
\hline & & & 0,66 & 54 & 0 & 1,72 & 0,89 & 3,00 & 0,98 & 2,02 & 0,82 & 3,12 & 1,00 & 2,12 & 0,85 & 1,75 & 0,58 & & 0,44 \\
\hline 24 & 0,80 & &, 72 & 67 & & & 0,91 & 2,54 & 0,82 & 1,72 & 0,72 & 3,12 & 0,98 & 2,140 & 0,88 & 1,89 & $0,62 \mid$ & 1,2 & 0,49 \\
\hline
\end{tabular}


Weit. Studien üb. die von einz. Organen hervorgebrachten Substanzen. II. 255 III. Gruppe.

Gemessen am 16. April 1917, 20 Tage nach der Einwirkung der Inkretstoffe. Rana temporaria.

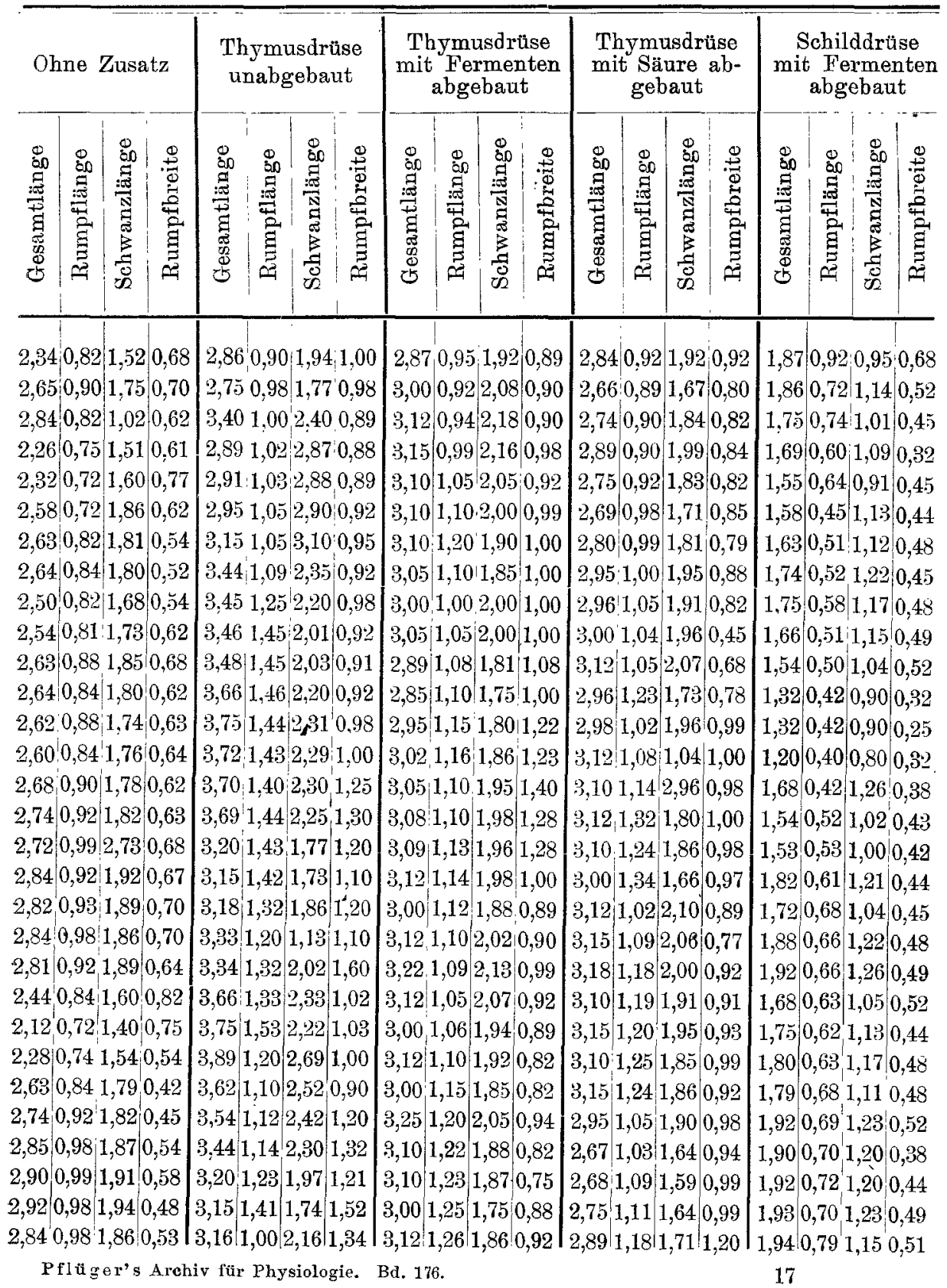


IV. Gruppe.

Gemessen am 15. Mai 1918, 14 Tage Dach Einwirkung der Inkretstoffe. Bufo vulgaris.

\begin{tabular}{|c|c|c|c|c|c|c|c|c|c|c|c|c|c|c|c|c|c|}
\hline \multicolumn{3}{|c|}{ Ohne Zusatz } & \multicolumn{4}{|c|}{$\begin{array}{c}\text { Thymusdrüse } \\
\text { unabgebaut }\end{array}$} & \multicolumn{4}{|c|}{$\begin{array}{c}\text { Thymusdrüse } \\
\text { mit Fermenten } \\
\text { abgebaut }\end{array}$} & \multicolumn{4}{|c|}{$\begin{array}{c}\text { Thymusdrüse } \\
\text { mit Säure ab- } \\
\text { gebaut }\end{array}$} & \multicolumn{3}{|c|}{$\begin{array}{c}\text { Thymusdrüse } \\
\text { Dialysat }\end{array}$} \\
\hline 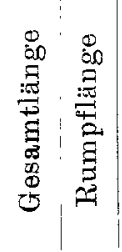 & 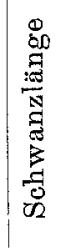 & 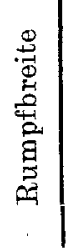 & 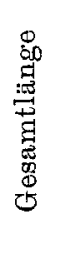 & 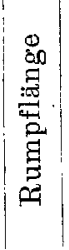 & 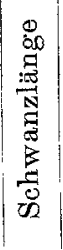 & 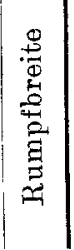 & 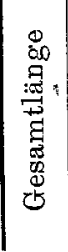 & 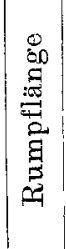 & 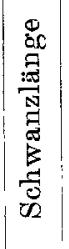 & 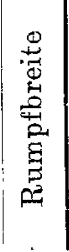 & 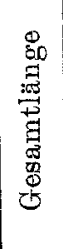 & 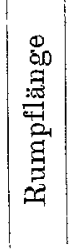 & 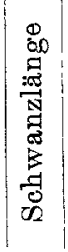 & 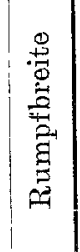 & 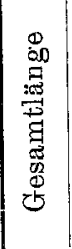 & 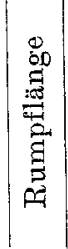 & 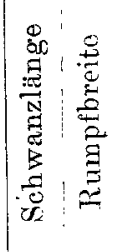 \\
\hline & & & & & 1,04 & 30 & 2,44 & 1,90 & 1,54 & 0.89 & 2,993 & $1,0,0$ & 1,90 & 30,91 & & & $(0,990,00$ \\
\hline 00 & 1,1 & 0,52 & 2,11 & 0,80 & 1,31 & 0,90 & 2,42 & 0,92 & 1,50 & 0,68 & 3,12 & 1,10 & 2 & 20,99 & & & $1,08^{i} 0,84$ \\
\hline $1,630,68$ & 1,0 & 0,50 & 2,12 & 0,72 & 1,40 & 0,80 & 2,44 & 0,85 & 1 , & 0,72 & 3,00 & 1,20 & & 0,94 & 1,99 & & $1,140,75$ \\
\hline $1,54 \mid 0,66$ & 60 & 0,50 & 2,48 & 0 & 1, & 0,80 & 2,48 & & & 0,77 & 3,00 & 1 & 1 & 50,85 & 1,98 & 8 & $1,100,80$ \\
\hline $1,620,58$ & 81, & 0,48 & 2,32 & 0 & 1 & 0,80 & 2,50 & 0 & 1 , & 0,70 & & 81 & & 00,82 & 78 & & 0,72 \\
\hline $1,62 \mid 0,54$ & 41, & 0,32 & 2,00 & 0 & 1 & 0,75 & 2,53 & & & 0,81 & & & & 0,84 & 1,89 & 0,80 & $1,090,71$ \\
\hline $1,63: 0,48$ & 8,1 & 0,44 & 2,10 & & & 0,72 & - & & & 0,69 & & & & 20,84 & 1,90 & & 1 \\
\hline $1,68 \mid 0$ & 01 , & 0,45 & 2,00 & 0,72 & & 0,70 & 5.5 & & & 0,67 & & & & 0,82 & 1,75 & 0,75 & $1,00,0,60$ \\
\hline $1,69 \mid 0$ & 21 , & 0,46 & 2,1 & & 1 & 0,62 & 2,66 & 0 & 1 & 0,70 & 2 & 0 & 1 & 00,75 & 1,80 & & 1 \\
\hline $1,710,51$ & 11 & 0,42 & 2,1 & 30,68 & 1 & 0,60 & 2,10 & 0 & & 0,65 & & & & 50,82 & 2,12 & 2 & $1,40^{\prime} 0,62$ \\
\hline $1,29,0,62$ & 20 & 0,41 & 1 , & & & 0,52 & 2,12 & 0 & & 0,68 & 2 & 9 & 1 & 10,80 & 2 & & 0,60 \\
\hline $1,86,0,68$ & & 0,48 & 1 , & & 1,27 & 0,50 & 2,14 & 0,79 & 1 & 0,70 & 32 & & & 20,85 & & & 0,62 \\
\hline $1,920,62$ & 21 , & 0,42 & 2,44 & & 1,56 & 0,50 & 2,18 & & & 0,70 & & & 1 & 70,75 & 2,16 & 6 & $1,500,61$ \\
\hline 1,7 & 11, & 0,48 & 2 & & 1,80 & 0,75 & 2,20 & 0 & & 0,71 & 10 & 30 & & 0,77 & 2,46 & 0,70 & $1,760,6: 3$ \\
\hline $1,820,60$ & 01 & 0,45 & 2,77 & & 1,88 & 0,78 & 2,18 & 0,72 & & 0,80 & & & 1,99 & 30,72 & 2,13 & 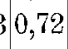 & $1,410,63$ \\
\hline $1,8, \mid 0,60$ & 01 , & 0,48 & 2 & & & 0,90 & 2,00 & 0,70 & 1,30 & 0,90 & 15 & 82 & {$[1,39$} & 90,70 & 1,98 & & 0,62 \\
\hline 1, & 0,1 , & 0,50 & 2,4 & & 91,55 & 0,72 & 1,98 & 0,70 & & 0,92 & 2,00 & 0,80 & 1,20 & 00,71 & 1,75 & 50,75 & $1,000,66$ \\
\hline 1,820 & & 0,45 & 24 & & 41,58 & 30,74 & 1,78 & & 50 & 0,82 & 00 & 5 & & 50,70 & 1,60 & 88 & $0,820,64$ \\
\hline 1,840, & 11, & 0,40 & 2,4 & 10 & 1 & 10,70 & 1,99 & 90 & & 0,88 & & & 1 & 80,65 & 1,78 & 30,80 & $0,980,68$ \\
\hline $1,920,62$ & & 0,51 & 2,40 & & & 0,72 & 2,14 & & 1,33 & 0,84 & 7 & 2 & 1 & \begin{tabular}{|l|l|}
5 & 0,62
\end{tabular} & 1,80 & 0,82 & $0,98,0,67$ \\
\hline $1,82,0,60$ & 01 , & 0,50 & 2,2 & & 41 & 80,73 & 2,14 & 40 & & 0,82 & 2,11 & 1 & 1,40 & $0,0,63$ & 1,75 & 50,82 & $0,930,68$ \\
\hline $1,88 \mid 0,62$ & 21 , & 0,42 & 2,3 & & 4 & 80,71 & 2,15 & & 51 & 0,88 & 2,0 & 75 & 51 & 00,68 & 1,89 & 0,85 & $51,040,67$ \\
\hline $1,920,68$ & 31 , & 0,43 & 2,1 & $2 \mid 0$ & 01 & 20,78 & 2,18 & 80,88 & 81,30 & 0,90 & 2,18 & 80 & 1,46 & 60,80 & 1,90 & 0,83 & $1,070,72$ \\
\hline & 41 & 0,48 & 2,1 & 40 & 51 & 90,70 & 2,12 & & & 0,92 & 2,12 & 0,76 & 61,36 & 60,81 & 2,10 & 0,84 & $|1,26| 0,78$ \\
\hline $1,810,66$ & 61,1 & 50,51 & 2,18 & & 0 & 80,70 & 2,48 & 80,88 & 81,60 & 0,90 & 2,18 & 80,74 & & $4 \mid 0,72$ & 2,15 & 50,82 & $21,23 \mid 0,82$ \\
\hline $1,810,62$ & 1 & 0,42 & 1 & & 1,48 & 80,78 & 2,32 & 0,84 & 41 & 8,00 & 2,48 & 80,80 & 01,68 & 80,69 & 2,14 & 40,88 & \begin{tabular}{l|l|l|l}
3 & 1,26 & 0,90
\end{tabular} \\
\hline $5,0,50$ & 01,2 & 0,48 & 2,2 & 00 & 01,50 & 0,72 & 2,22 & 20,82 & 1,40 & 0,70 & 50 & 0,82 & 1,68 & 80,68 & 2,10 & $0,0,90$ & $1,20,0,90$ \\
\hline $1,80,0,52$ & 21,2 & 0,44 & 2,1 & 40,71 & 11,43 & 8,70 & 2,25 & $5 \mid 0,84$ & 41,41 & 0,60 & 2,18 & 80,80 & 01,38 & 80,80 & 2,12 & 20,60 & $1,52: 0,70$ \\
\hline $1,88,0,63$ & 1,2 & 0,44 & 2,1 & 20,71 & 1,41 & 0,69 & 2,00 & 0,81 & 1,19 & 0,94 & 2,12 & 0,78 & 81,34 & 40,92 & 2,14 & $4: 0,67$ & $7,47: 0,72$ \\
\hline
\end{tabular}


Weit. Studien üb. die von einz. Organen hervorgebrachten Substanzen. II. 257

Wachstums, wobei gleichzeitig die Metamorphose zum Teil gehemmt war. In anderen Fällen war die Metamorphose sehr stark beschleunigt. Man hatte in vielen Fällen den Eindruck einer Thymuswirkung kombiniert mit Schilddrüsenwirkung, wobei bald die eine Wirkung, bald, die andere überwog. Zum Teil war das Grössenwachstum ein ganz ausserordentliches. Bei vier von zehn Riesentieren war eine stark vergrösserte Hypophyse vorhanden. Die gleiche Beobachtung wurde an drei spontan zu auffallend grossen Tieren entwickelten Kaulquappen von Rana esculenta gemacht. ${ }^{1}$ ) Die übrigen sieben der erwähnten zehn Riesenlarven hatten keine vergrösserte Hypophyse. Manchmal traten auch hier Missbildungen auf. Sie sind in der Tafel V (Abb. 117 a) dargestellt. Wie schon erwähnt, waren die Resultate ungleich. In manchen Fällen war überhaupt keine besondere Wirkung feststellbar. Die Versuche können zurzeit nicht als abgeschlossen betrachtet werden. Es muss eine Trennung der bekannten drei funktionell so verschiedenen Teile der Hypophyse angestrebt werden. Es ist wohl möglich, dass das unterschiedliche Verhalten darauf zurückzuführen ist, dass alle drei Drüsenanteile zugleich aber in verschiedenem Ausmasse zur. Wirkung gekommen sind.

\section{Einfluss von Geschlechtsdrüsensubstanz auf Wachstum und Entwicklung.}

Wir haben einerseits Hodensubstanz auf ihre Wirkung auf Kaulquappen geprüft, und ferner aus Ovarien gewonnene Produkte. Bei den letzteren haben wir zum Teil die Corpora lutea getrennt von der übrigen Eierstocksubstanz untersucht. Die Ergebnisse waren leider auch hier keine so gleichmässigen wie beiden Schilddrüsen-und Thymusdrüsensubstanzversuchen Bei der Verwendung von Hoden fanden wir in den meisten Fällen ein rascheres Wachstum, währond die Metamorphose nicht wesentlich beeinflusst schien. Vgl. Tafel V, Abb. 120-122.

Besonders unregelmässig waren die Resultate mit aus Ovarjen gewonnenen. Substanzen. Auffallend häufig erhielten wir kleine, zum Teil missgestaltete Tiere. Sie glichen zum Teil den Schilddrüsentieren. indom die Entwicklung überstürzt verlief, doch waren in Einzelheiten Unterschiede vorhanden, wie schon ein Vergleich der Abbildungen auf Tafel V, Abb. 123-128 zeigt.

\section{Einfluss von Nebennierensubstanzen auf Wachstum und Entwicklung.}

Die Nebennierentiere fielen meist schon dadurch auf, dass sie beständig in ausserordentlich lebhafter Bewegung waren. Sie blieben

1) Vgl. auch hierzu Amandus $\mathrm{Hahn}$, Arch. f. mikrosk. Anat. Bd. 80 Abt. I. 1. 1912. 
zum Teil sehr kleín. Bei einer Reihe von Tieren trat ein eigenartiges Phänomen auf, das auf Tafel $V$ in Abbildung 129-32 dargestellt ist. Zunächst bemerkte man, dass die äussere Haut sich abzuheben begann. Nach einiger Zeit erblickte man den Körper der Tiere in der Tiefe einer dünnen, mit wasserklarer Flüssigkeit erfüllten Blase. Die Tiere lebten in diesem Zustande bis 6 Wochen. Es hatte sich offenbar ein Transsudat gebildet, das die Haut am ganzen Körper abgehoben hatte. Es ist naheliegend, an eine Wirkung des Adrenalins auf die Blutgefässe zu denken. Wir konnten allerdings bis jetzt denselben Zustand mit Adrenalin selbst nicht hervorrufen. Erwähnt sei ierner, dass mehrfach bei Schilddrüsen- und Thymustieren ganz ähnliche Erscheinungen zu heobachten waren.

\section{Einfluss von Plazentasubstanz anf Wachstum und Entwicklung.}

Verfütterung von Plazentagewebe wirkte ausserordentlich heschleunigend auf die Entwicklung von Kaulquappen. Es entstanden innerhalb weniger Tage vollentwickelte Fröschehen bzw. Krötchen. Im Gegensatz zur Schilddrüsensubstanz-Wirkung war die rasche Entwickluing nicht mit einer starken Steigerung des Verbrauchs von Körperstoffen verknüpft. Während die typischen Schilddrüsentiere „Geigenform" annehmen, das heisst besonders im hinteren Teil des Körpérs auffallend schmal sind, war das bei den Plazentatieren nicht der Fall. Sie entwickelten sich zu ganz normal aussehenden Tieren. Abgebaute Plazenta hatte qualitativ die gleiche Wirkung, nur war die Beschleunigung des Wachstums nicht so bedeutend. Versuche, durch Auszüge mit organischen Lösungsmitteln die wirksamen Substanzen zu isolieren, sind auch hier ausgeführt worden. Über die erhaltenen Resultate soll berichtet werdèn, sobald mehr Eriahrungen vorliegen.

Wir haben weiterhin noch Versuche mit aus Pankreas und Nieren gewonnenen Stoffen durchgeführt, und ferner geprüft, ob nicht vielleicht Abbaustufen aus Organen, die innensekretorisch vorläufig noch wenig in Frage gezogen sind, wie Muskeln, Haut, Lunge, Milz usw. auch bestimmte Wirkungen auf die Kaulquappen ausüben. Es war dies nicht der Faill. Ferner haben wir Peptone der verschiedensten Abkunft (aus Haaren, Federn, Seide usw.) auf Kaulquappen einwirken lassen, um zu sehen, ob nicht vielleicht ähnliche Erscheinungen hervorgerufen werden, wie bei der Anwendung der oben beschriebenen, spezifisch wirkenden Organsubstanzen. Wir führten cine grosse Anzahl derartiger Versuche durch, um uns zu überzeugen. ob wirklich spezifische Wirkungen den beobachteten Er- 
Weit. Studien üb. die von einz. Organen hervorgebrachten Substanzen. II. 259

gebnissen zugrunde liegen. Diese Peptnne vermochten züm Teil wohl Hemmungen in der Entwicklung zu bewirken, jedoch wurlen keine „Thymustypen" erhalten.

Eine sehr grosse Anzahl von Untersuchungen war der Frage gewidmet, ob es möglich ist, durch Kumbination verschiedener Organsubstanzen eine besondere Wirkung zu entfalten. Wir hatten die Hoffnung, auf diesem Wege das Zusammenspiel der Inkrete studieren zu können. Zu diesen Versuchen verwandten wir ausschliesslich die vollständig abgebauten Organe, sei es, dass sie mit Hilfe von Fermenten, sei es mit Hilfe verdünnter Schwefelsäure zerlegt worden waren. Es ist sehr schwer, aus den erhaltenen Resultaten irgend welche Schlüsse zu ziehen. Fs zeigte sich immer wieder, dass im besonderen die Thymus- und Schilddrüsenwirkung überall da überwiegt, wo Substanzen aus diesen Organen zur Anwendung kamen. Eine Reihe von Abbildungen auf der Tafel VI mag einige dieser Ergebnisse illustrieren. Es handelt sich ausschliesslich um Tiere, die 14 Tage unter der Wirkung der betreffenden Substanzen gestanden hatten. Die abgebildeten Tiere sind alle gleichalterig.

Erwähnt seien schliesslich noch einige Versuche, die mit Sarkomen und Karzinomen ausgeführt worden sind, und zwar verwendeten wir zum Teil unabgebaute, zum Teil abgebaute Gewebe. Wir haben dabei mehrfach Störungen in der ganzen Entwicklung beobachtet. Die Zahl der Versuche ist jedoch zu klein. Tabelle V (Abb. 135-139) zeigt einige Resultate dieser Versuche. Wir baben ferner auch Hefesubstanz auf ihre Wirksamkeit auf Wachstum und Fntwicklung von Kaulquappen geprüft. Es zeigte sich, dass die letztere beschleunigt wird, jedoch in der Hauptsache nur in den ersten Tagen.

Wenn wir alle Ergebnisse zusammenfassen, dann kommen wir zu dem Resultat, dass unzweifelhaft bewiesen ist, dass man bei Kaulquappen regelmässig mit bestimmten Organsubstanzen einen ganz spezifischen Einfluss auf das Wachstum und die Entwicklung gewinnen kann. Am ausgesprochensten und regelmässigsten sind die Erscheinungen bei Schilddrüsen- und Thymussubstanz. Typische Erscheinungen sahen wir ferner, jedoch nicht regelmässig, bei Verwendung von Hypophysen-, Hoden-, Ovarien- und Nebennierensubstanz. Bei Verwendung von Hypophysen- und Hodensubstanz beobachteten wir ein verstärktes Wachstum. Die Entwicklung verhielt sich dabei unregelmässig. Bei Verwendung von Ovariensubstanz erhielten wir im allgemeinen schmale und lange Tiere mit beschleunigter Entwicklung. Durch Kombination der aus verschiedenen Organen gewonnenen Substanzen erzielten wir ganz deutliche Beeinflussungen der einzelnen wirksamen Stoffe. Meist überwiegt die Wirkung der einen Substanz. Oft gelingt es aber auch, eine kombinierte 
Wirkung zu beobachten. So erhält man mit Thymusdrüse allein starkes Wachstum und verlangsamte Entwicklung. Wendet man Thymussubstanz und Schilddrüsensubstanz in geeigneter Kombination an, dann erhält man eine raschere Entwicklung und gleichzeitig auch vermehrtes Wachstum. Würden wix die Inkretstoffe kennen, dann könnten wir sie genau dosieren und dam auch die Mengenverhälinisse, unter denen sie die eine oder andere Wirkung zeigen, ganz genau angeben.

Das bemerkenswerteste Ergebnis der ganzen Forschung ist das, dass die beobachteten Erscheinungen aufgetreten sind, gleichgültig, ob wir die Organe selbst verwandten oder aber die durch Hydrolyse aus ihnen gebildeten Produkte. Damit ist bewiesen, dass die wirksamen. Stoffe einfacherer Natur sein müssen. Im allgemeinen ist der Einfluss der nicht abgebauten Organe quantitativ demjenigen der gleichen Menge abgebauten Gewebes überlegen.

Die aasgeführten Untersuchungen bedürfen noch weiterer Ausarbeitung. Es muss vor allen Dingen in exakter Weise festgestellt werden, welche morphologische Wirkung, durch die einzelnen Inkretstoffe hervorgerufen werden. Wir haben bis jetzt die Tiere in der Hauptsache beobachtet und gemessen. In Zukunft werden wir die einzelnen organischen Veränderungen genau zu studieren haben. Versuche in dieser Richtung sind auf braiter Grundlage bereits im Gange ${ }^{1}$ ).

Erwähnen möchten wir, dass auch Versuche mit den entsprechenden Kaltblüterorganen ausgeführt worden sind. Wegen der Kleinheit der betreffenden Organe ist bis jetzt ein sicheres Ergebnis noch nicht gewonnen.

\section{Erklärung der Tafeln.}

\section{Tafel III.}

Abb. 1-72: Schilddrüsentiere. Die Tiere 1 und 2 erhielten unabgebaute Schilddrüse. $\quad 3-6$ wurden mit verdauter Schilddrüsensubstanz gefüttert. $7-15$ erhielten mit verdünnter Schwefelsäure bereitete Abbauprodukte aus Schilddrüse. Die Abb. 16-30 zeigen ältere Stadien von Schild drüsentieren (Abb. 27-30 sind um die Hälfte vergrössert).

In $\mathrm{Abb} .81 \mathrm{a}$ und $\mathrm{b}$ sind $\mathrm{Ax}$ olotl wiedergegeben; a ist das Schilddrüsen-, b das Kontrolltier.

Abb. 32-47: Schilddrüsentiere (Kaulquappen von Bufoarten).

Abb. 48-51 normale Tiere (Kontrolltiere).

Abb. 52-68: Schilddrüsentiere. Verabreicht wurde Dialysat von anverdauter Schilddrüse.

Abb. 69-72: gleichaltrige $\mathrm{K} \circ \mathrm{n}$ trolltiere.

Abb. 73-80: Strumatiere.

1) Vgl. hierzu auch die wichtigen Studien von B. Romeis, Zeitschr. f. die gesamte experim. Med. Bd. 5 S. 99. 1916 u, Bd. 6 S. 101. 1918. 
Weit.Studien üb. die von einz. Organen hervorgebrachten Substanzeu. I1. 261

\section{Tafel IV.}

Abb. 81-82: Strumatiere (Fortsetzung von Tafel III).

Abb. 84 -88: Schilddrüsentiere. Einwirkung grösserer Mengen von. abgebauter Schilddrüsensubstanz. Abb. 89-105: Th ymustiere. Tiere 89-98 unter der Wirkung vollständig abgebauter Thymus in den ersten 4-6 Wochen. Tiere 99'-104 standen 2-6 Monate unter dem Einfluss dieser Produkte. a und b geben jeweilen zwei Ansichten des gleichen Tieres wieder.

Abb. 105 a zeigt ein Thymustier u. $105 \mathrm{~b}$ das gleichaltrige Kontrolltier. Abb. 106a und b stellen besondere Formen von Thymustieren dar. Abb. 107 b zeigt einen Axolotl unter Thymuswirkung. Tier $107 \mathrm{a}$ ist das gleichaltrige Kontrolltier. Tier 108 a ist das Kontrolltier zum Thymustier $108 \mathrm{~b}$.

\section{Tafel V.}

Abb. 109 b: Thymustier. Die beiden anderen Tiere (a) sind gleichaltrige, normal ernährte Tiere.

Abb. 110a: Thymustier, b Kontrolltier.

Abb. 111-115: a Hypophysentier und $\mathrm{b}$ das Kontrolltier.

Abb. 116: a Hypophysentier, b gleichaltriges Ovarientier, c Kontrolltier.

Abb. 117a: Hypoph ysentiere, Missbildungen.

Abb. 117: Kontrolltier zu 117 a.

Abb. 118: Grösstes Tier, Hypophysentier, die übrigen $\mathrm{Ab}$ - bildungen stellen normal ernährte, gleichaltrige Tiere dar.

Abb. 119a: normal ernährtes Tier, bund c Hyp ophysentiere. Abb. 120-122: a normal ernährte Tiere, b Hodentiere.

Abb. 123-128: Ovarientiere.

Abb. 129-132: Nebennierentiere. Die Abbildungen zeigen die eigenartige Ablösung der Haut.

Abb. 138-134: Weitere Nebennierentiere.

Abb. 185-138: Sarkomtiere.

Abb. 139a: Sarkomtier, b Kon-' trolltier.

\section{Tafel VI.}

Wirkung der Verabreichung von abgebauten Gewebssubstanzen aus mehreren Organen.

Abb. 140: Thymus-Schilddrüsentier, a Schilddrüsentier, b normales Tier.

Abb. 141: Verfütterung von Thymus + Schilddrüse.

$A b b .141 a$ : Verfutterung von Schilddrüse + Ovarium + Thymus + Hypophyse + Hoden.

Abb. 141 b: Verfütterung von Schild drüse + Hypophyse.

Abb. 142a: Ovarium + Hypophyse + Hoden verfüttert.

Abb. 142 b: Schilddrüse + Thy mus $+\mathrm{H}$ oden verfüttert.

Abb. 142c: Ovarium + Thymus verabreicht.

Abb. 143: Ovarium + Hoden + Hypophyse.

Abb.144a: Schilddrüse + Hypophyse.
Abb. 144b: Thymus + Hypophyse.

Abb. 144c: Hypophyse + Hoden. Abb. 144d: Thymus + Hoden.

Abb. 144e: Hypophyse + Thymus + Hoden.

Abb. 144f: Thymus + Schilddrüse + Hoden.

Abb. $144 \mathrm{~g}$ : Thymus + Ovarium.

Abb. 145a: Ovarium.

Abb. 145b: Schilddrüse.

Abb. $145 \mathrm{c}$ : Hypophyse.

Abb. 146: Thymus.

Abb. 146a: Thymus + Hypophyse. Abb. 146 b: Schilddrüse + H ypophyse.

Abb. 147a: Ovarium + Hypophyse.

Abb. 147b: Ovarium + Schilddrüse. 
Abb. 147c: Ovarium + Thymus. Abb.148a: Hy pophyse + Ovarium. Abb.148b: Hypophyse + Thymus. Abb.149a:Hyp ophyse+Thymus. Abb. 149 b: Hypophyse+Schild. dxüse.

Abb. 149c: Thymus.

Abb.150: Ovarium $+\mathrm{Hypophyse}$ + Hoden.

Abb. $151 a:$ Hypophyse + Schilddrü se.

Abb.151b:Hypophyse + Thymus.

Abb. 151c: Schilddrüse.

A.bb. 151 d: Thymus.

Abb. 152a: Struma.

Abb. 152b: Struma + Schilddrüse.

Abb. 158a: Thymus.

Abb. 153 b: Nebenniere.

Abb. $153 \mathrm{c}:$ H ypophyse + Hoden.

Abb. 153d: Hypophyse + Nebenniere.

Abb. 154: Hy pop hyse.
Abb. 154a: Ovarium.

Abb. 155a: Hoden.

Abb. 155b: Schilddrüse.

Abb. 155c: Nebenniere.

Abb. 155d: Kontrolltier.

Abb. 156a: Hy pophyse.

Abb. $159 \mathrm{~b}$ : Hoden.

Abb. $156 \mathrm{c}$ : Schilddrùse.

Abb. 156d: Nebenniere.

Abb. 157a: Thy mus.

Abb. 157 b: Schilddrüse.

Abb. 158a: Hypophyse+Ovarium. Abb. 158b: Scbilddrüse + Ovarium.

Abb. 158c: Schilddrüse.

Abb. 159a: Schilddrüse + Hypophyse.

Abb. 159b: Thymus + Hypophyse. Abb. 159c: Schilddrüse + Hoden. Abb. 159d: Thymus + Ovarium. Abb. 159d: Thymus + Ovarium. Abb. 159 e: Thymus.

Versuche an Axoloteln.

Abb. 160 a: Hypophyse. Abb. 160b: Nebenniere.
Abb. 161: Hoden.

Abl. 162: 'Thymus. 


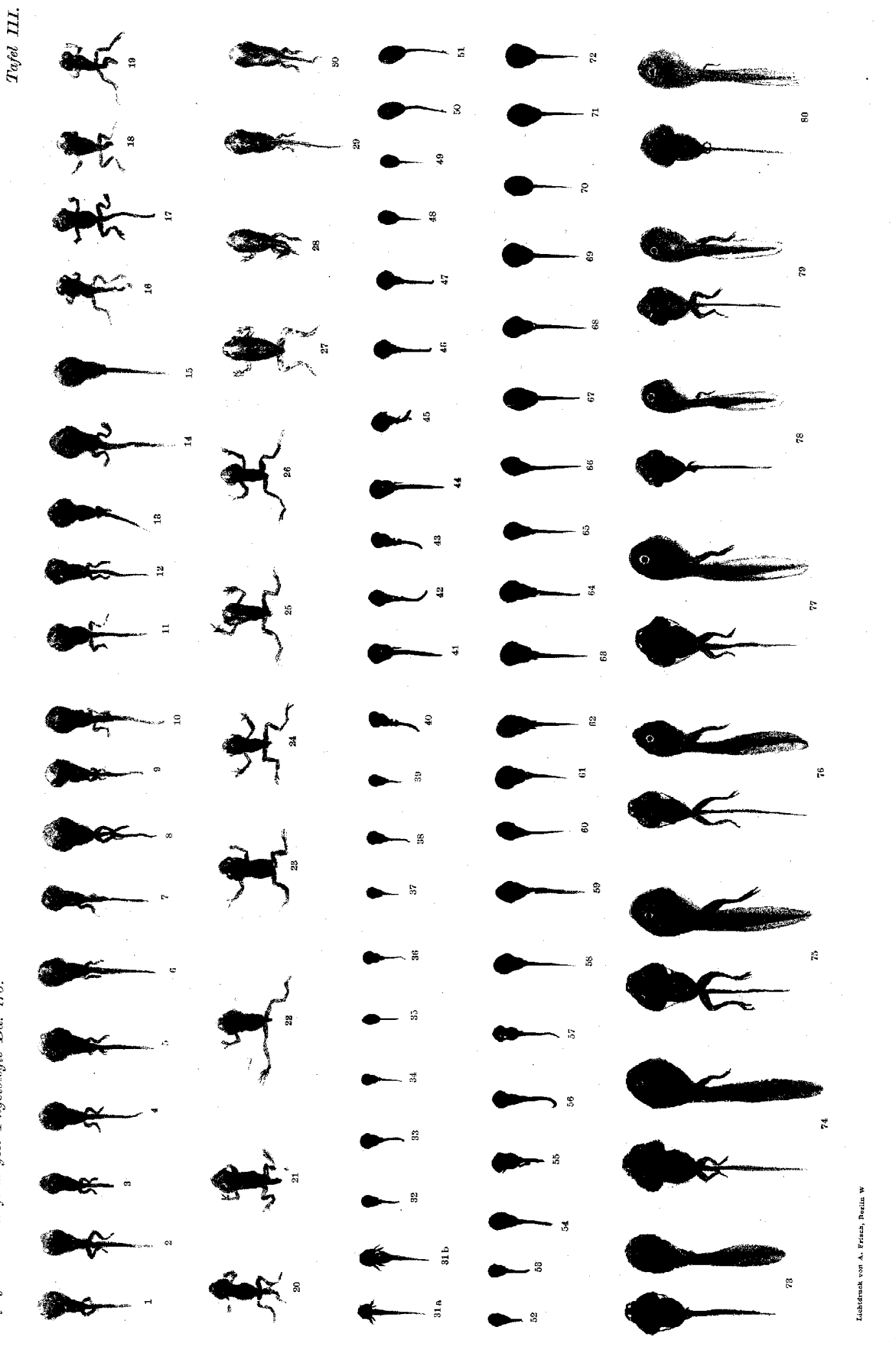


3
है

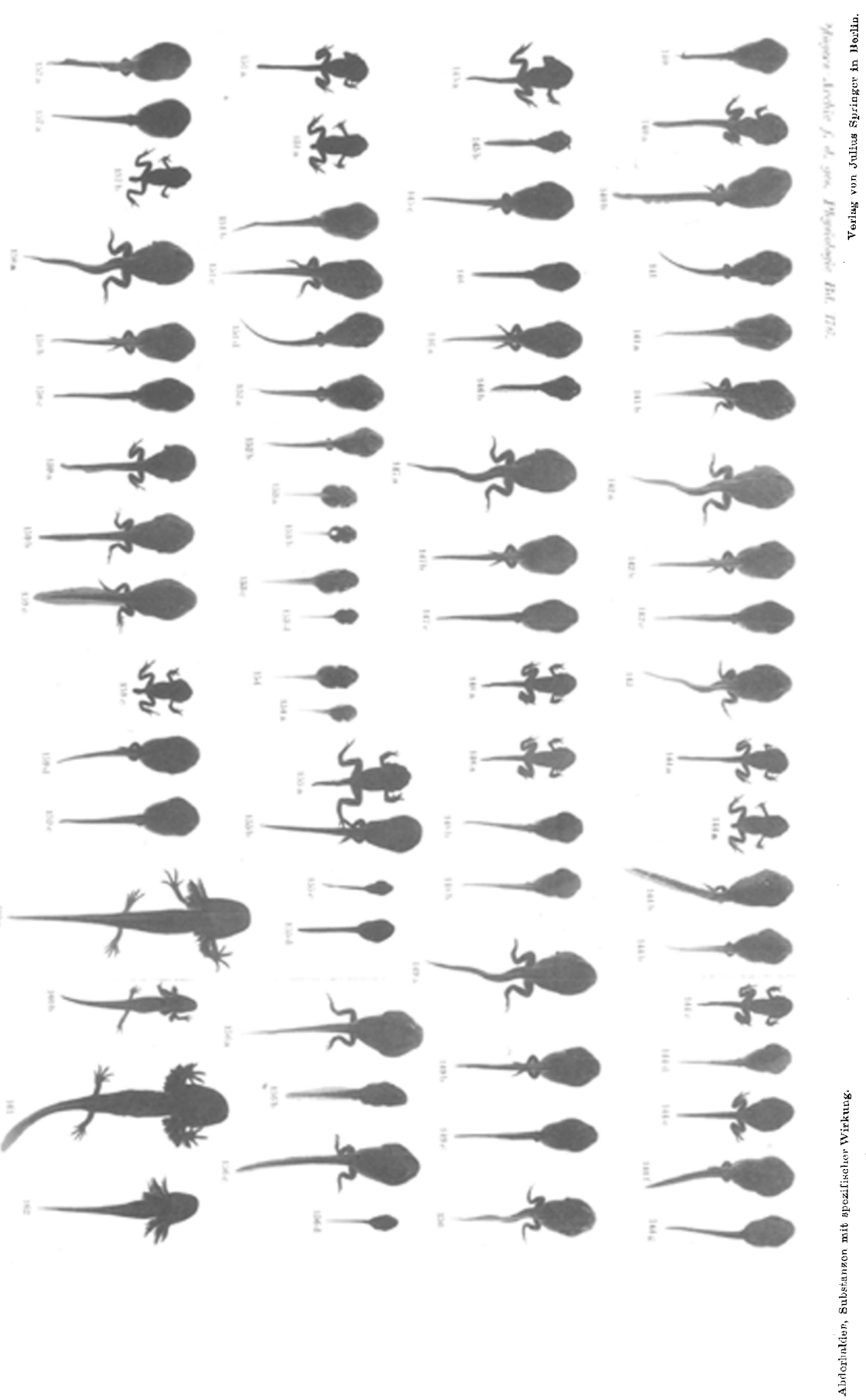

5

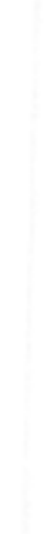

$\dot{0}$
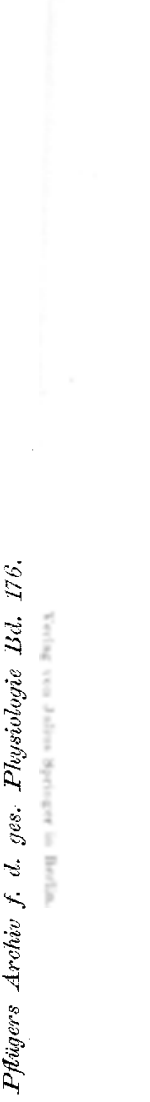
$\frac{2}{2}$

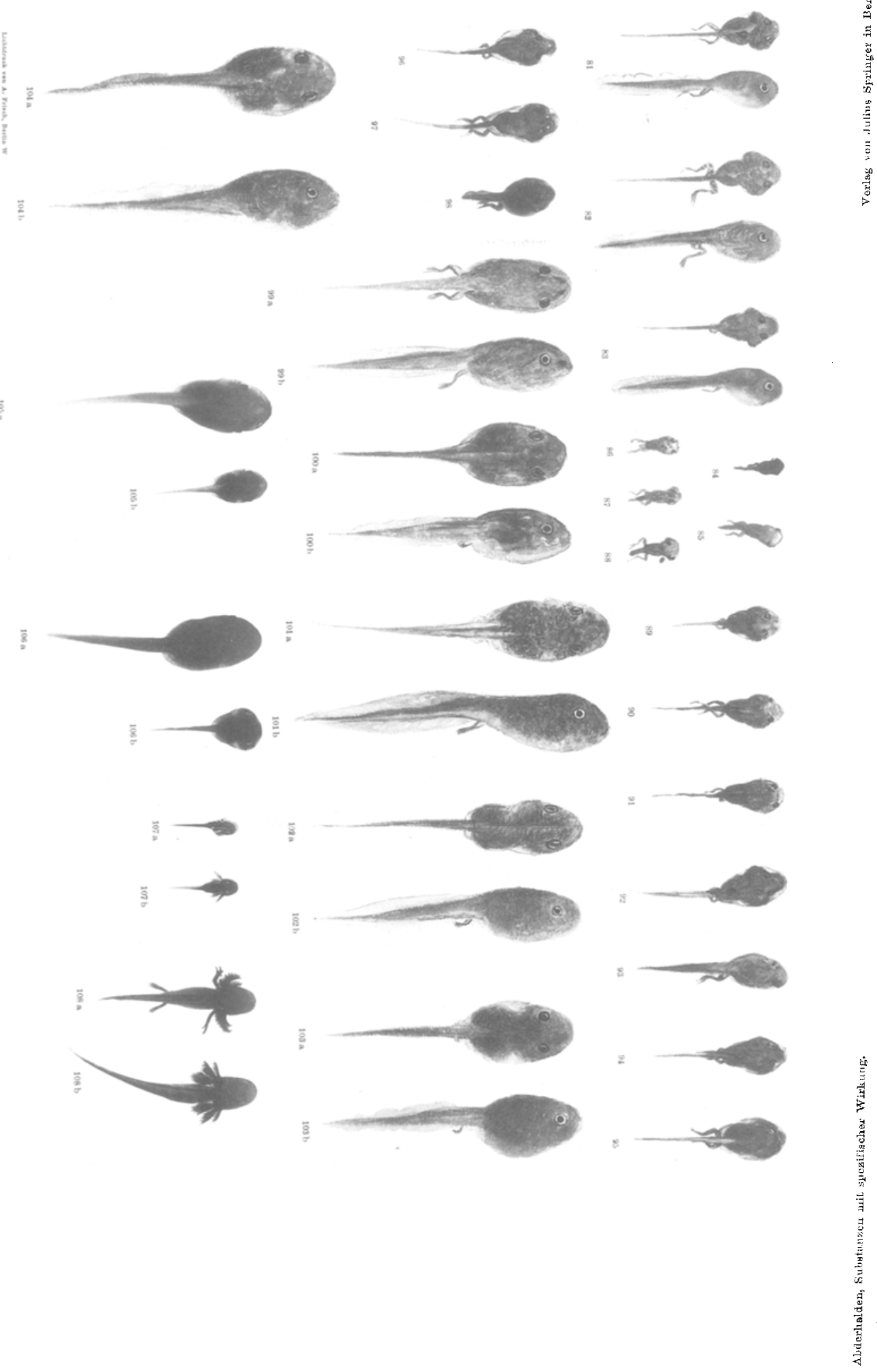


$\frac{1}{3}$

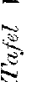

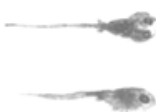

$\frac{1}{12}$
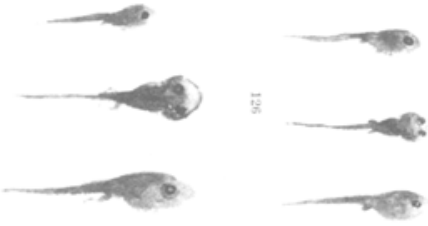

$-6$

$\longrightarrow$

E
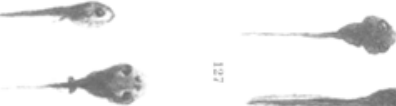

땅
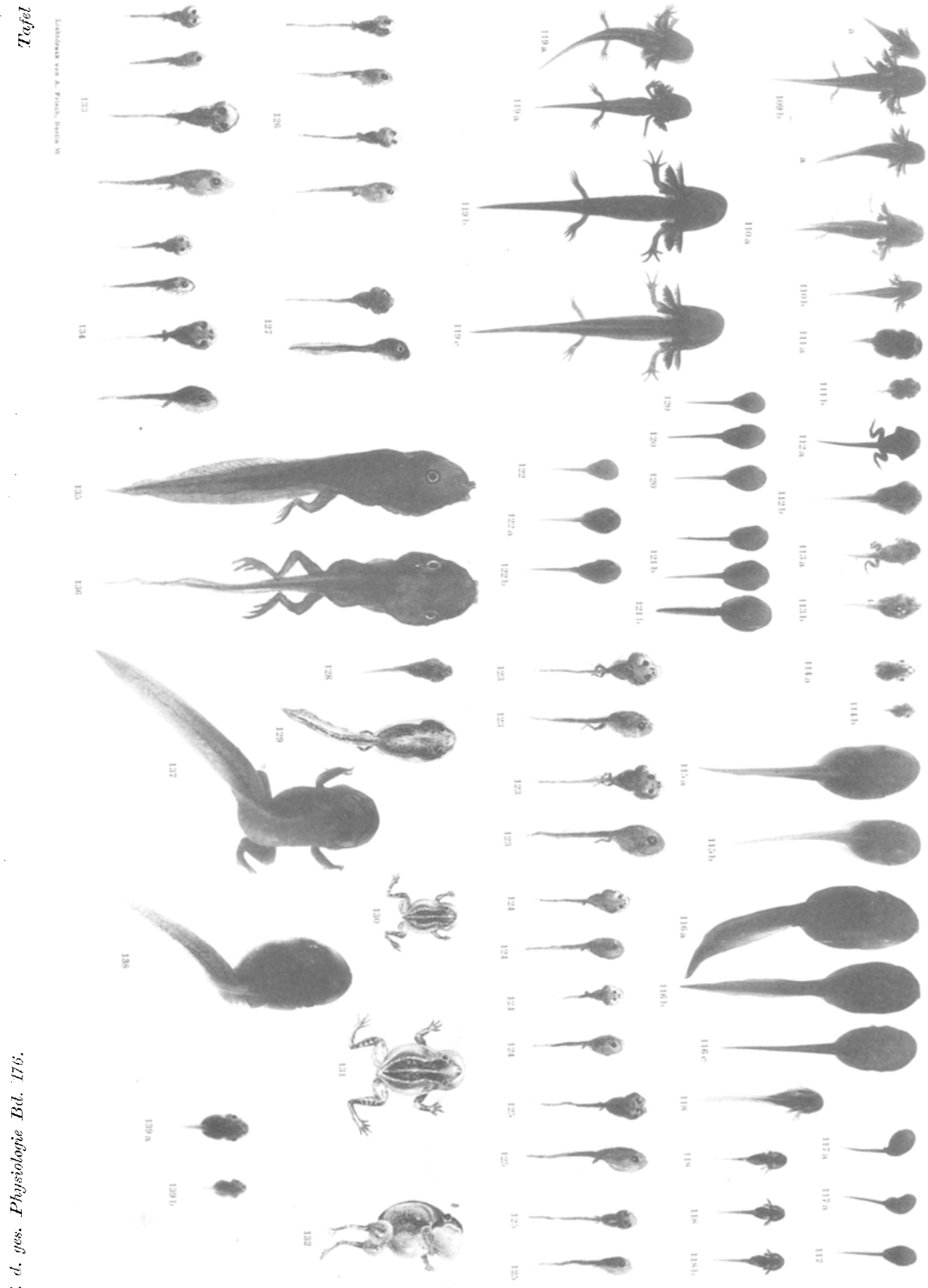